\title{
Antibiotic treatment induces activation of microglia and impairment of cholinergic gamma oscillations in the hippocampus
}

Gürsel Çalışkan ${ }^{1,2, *}$ \#, Timothy French ${ }^{3,}$, Markus M. Heimesaat ${ }^{4}$, Ildiko Rita Dunay ${ }^{2,3, \dagger}$,

Oliver Stork ${ }^{1,2, \dagger}$

${ }^{1}$ Institute of Biology, Otto-von-Guericke University, Magdeburg, Germany

${ }^{2}$ Center for Behavioral Brain Sciences, Magdeburg, Germany

3 Institute of Inflammation and Neurodegeneration, Medical Faculty, Otto-von-GuerickeUniversity, Magdeburg, Germany

${ }^{4}$ Institute of Microbiology, Infectious Diseases and Immunology, Charité - University Medicine Berlin, Berlin, Germany

Short Title: Antibiotics, immune function and hippocampal physiology

\section{\#Shared first authorship}

${ }^{\dagger}$ Equally contributed last authorship

${ }^{\star}$ Corresponding Author

Dr. Gürsel Çalışkan

Institute of Biology

Otto-von-Guericke-University

Leipziger Str. 44, Haus 91

Magdeburg, 39120, Germany

Tel: +49-391-6755108

Fax: +49-391-6755102

E-mail: guersel.caliskan@ovgu.de

Keywords: Antibiotics, immune function, microglia, hippocampus, synaptic transmission, gamma oscillations

Word

count:

7259

(including

figure

legends) 


\section{$1 \quad$ Abstract (251 words)}

2 Antibiotics are widely applied for the treatment of bacterial infections, but their long-term use

3 may lead to gut flora dysbiosis and detrimental effects on brain physiology, behavior as well

4 as cognitive performance. Still, a striking lack of knowledge exists concerning

5 electrophysiological correlates of antibiotic-induced changes in gut microbiota and behavior.

6 Here, we investigated changes in the synaptic transmission and plasticity together with

7 behaviorally-relevant network activities from the hippocampus of antibiotic-treated mice. The

8 prolonged antibiotic treatment led to a strong reduction of myeloid cell pools in bone marrow,

9 circulation and those surveilling the brain. Upon antibiotic treatment, circulating Ly6C $\mathrm{C}^{\text {hi }}$

10 inflammatory monocytes adopted a proinflammatory phenotype with increased expression of

11 CD40 and MHC II. In the central nervous system, microglia displayed typical signs of

12 activation with elevated CD40 and MHC II expression, as well as increased IL-6 and TNF

13 production. Concomitantly, we detected a substantial reduction in the synaptic transmission

14 in the hippocampal CA1 after antibiotic treatment. In line, carbachol-induced cholinergic gamma oscillation were reduced upon antibiotic treatment while the incidence of hippocampal sharp waves was elevated. These changes were associated with the global changes in the expression of neurotrophin nerve growth factor and inducible nitric oxide synthase, both of which have been shown to influence cholinergic functions. Overall, our study demonstrates that antibiotic-induced changes in the gut microbiome and immune cell function are associated with a drastic reduction in the synaptic transmission and gamma oscillations in the hippocampus, a brain region that is critically involved in mediation of innate and cognitive behavior. 


\section{Introduction}

Millions of humans are being prescribed antibiotics (Abx) every day (Suda et al., 2014). However, accumulating evidence from human and rodent studies indicate that the long-term use of antibiotics can lead to detrimental effects on hematopoiesis, brain physiology, innate behavior, and cognitive functions (Desbonnet et al., 2015; Fröhlich et al., 2016a; Josefsdottir et al., 2017; Möhle et al., 2016b). This is due, at least in part, to the decimation of microbiota that colonize our intestinal tract and the disruption of normal gut microbiota-to-brain signaling.

Converging evidence points toward a critical role of microbiota in regulation of the crosstalk between peripheral immune cells and the central nervous system (CNS) (Baruch et al., 2015; Schwartz et al., 2013). We have previously shown that prolonged antibiotic-induced microbial depletion reduces hippocampal neurogenesis and memory retention. Moreover, we demonstrated that these changes are partially mediated by circulating Ly $6 \mathrm{C}^{\text {hi }}$ monocytes as adoptive transfer of these cells from naïve mice could rescue the observed neurogenesis and memory decreases (Möhle et al., 2016b). Monocytes and monocyte-derived macrophages comprise a fundamental leukocyte subset of the innate immune response with multifaceted roles in maintenance of host tissue homeostasis (Biswas et al., 2015; Hammond et al., 2014; Möhle et al., 2016a). Depending on the environment, infiltrating monocytes and CNS resident microglia support diverse functions ranging from inducing to resolving neuroinflammation (Cardona et al., 2006; Michell-Robinson et al., 2015; Shechter and Schwartz, 2013). However, the details of this crosstalk in the homeostatic regulation of normal CNS function remains largely elusive.

One hub region mediating distinct aspects of cognitive function as well as affective behavior is the hippocampus (Çalışkan and Stork, 2018; Maren et al., 2013; Möhle et al., 2016b). Changes in the synaptic transmission and plasticity in hippocampal circuitries have been studied for decades as correlates of these functions (Citri and Malenka, 2008). Indeed, abnormal hippocampal synaptic transmission or plasticity has been reported in numerous neuropsychiatric, neurodegenerative and/or neurodevelopmental conditions with aberrant neurocognitive functions (Annamneedi et al., 2018; Connor et al., 2011; Maggio and Segal, 2011; Rowan et al., 2003). Despite the convincing evidence for the importance of healthy/intact gut microbiota for normal emotional and executive behavior (Bercik and Collins, 2014; Desbonnet et al., 2015; Heijtz et al., 2011; Mayer, 2011; Möhle et al., 2016b; Mostafa and Miller, 2014; Neufeld et al., 2011; Sarkar et al., 2020), to date, there is no direct evidence for the impact of antibiotic-induced gut dysbiosis on hippocampal synaptic transmission and its plasticity. 
The hippocampus generates diverse oscillatory rhythms that represent distinct behavioral and cognitive states (Buzsaki, 2004). Specifically, gamma oscillations $(30-100 \mathrm{~Hz})$ serve as a common oscillatory mechanism not only for memory encoding/retrieval (Fell and Axmacher, 2011; Montgomery and Buzsaki, 2007), but also for affective behaviors such as fear and anxiety (Headley and Paré, 2013). In the hippocampus, generation and sustainment of gamma oscillations are strongly dependent on cholinergic level both in vitro and in vivo (Caliskan et al., 2015; Fisahn et al., 1998; Vandecasteele et al., 2014). Of note, neurotrophic factors such as nerve growth factor (NGF) are important regulators of cholinergic activity and potentially can influence hippocampal gamma oscillations (Conner et al., 2009). In addition, microglia activity and glia-associated factors such as tumor necrosis factor (TNF) and inducible nitric oxide synthase (iNOS) can modulate hippocampal synaptic transmission or gamma oscillations (Adaikkan and Tsai, 2020; Beattie et al., 2002; laccarino et al., 2016; Martorell et al., 2019; Papageorgiou et al., 2016; Ta et al., 2019). On the other hand, under low cholinergic tonus, hippocampal circuits generate sharp wave-ripples both in vivo and in vitro (Buzsáki, 2015; Maier et al., 2003). These events may represent on-going plasticity in hippocampal circuitries (Çalışkan and Stork, 2018) and are associated with successful memory consolidation (Girardeau and Zugaro, 2011); pathological alterations in their incidence have been observed in association with abnormal memory formation (Çalışkan et al., 2016; Polepalli et al., 2017). To our knowledge, however, no studies have investigated the impact of antibiotic-induced gut dysbiosis on these behaviourally relevant hippocampal network activities to date.

Given the profound impact of antibiotic treatment on cognitive performance and affective behavior (Desbonnet et al., 2015; Fröhlich et al., 2016b; Möhle et al., 2016b), we hypothesized that antibiotic-induced gut dysbiosis might be associated with aberrant changes in the hippocampal synaptic physiology and associated brain rhythms. Accordingly, we elucidated several factors associated with peripheral and central immunoregulation, cytokine and neurotrophin levels in the CNS, and investigated hippocampal synaptic transmission as well as network oscillations upon antibiotic treatment. Our study indicates that gut dysbiosis is associated with strong alterations in the peripheral and CNS immune function and provides first insights into the potential impact of gut dysbiosis on hippocampal synaptic transmission and behaviorally-relevant hippocampal network activities. 
91

\section{Methods}

\subsection{Animals}

C57B/6JBomTac mice were obtained from M\&B Taconic, Germany, and bred in the animal facility at the Otto-von-Guericke University Magdeburg (12h light/dark cycle with lights switched on at 19:00 $\mathrm{hr}$ with a $30 \mathrm{~min}$ dawn phase; food and water ad libitum). All experiments were conducted in accordance with the European and German regulations for animal experiments and were approved by the Landesverwaltungsamt Saxony-Anhalt (Permission Nr. 203.h-42502-2-887 OvGMD; 203.h-42502-2-1206 UniMDOVGU).

\subsection{Antibiotic Treatment and microbiota assessment}

Male mice were group-housed ( 3 to 6 mice per cage) and treated with broad-spectrum antibiotics (Abx) according to the protocol previously published (Möhle et al., 2016b). The Abx compounds consisting of ampicillin plus sulbactam (1.5 $\square \mathrm{g} / \mathrm{L}$; Pfizer), vancomycin (500 $\mathrm{mg} / \mathrm{l}$; Cell Pharm), ciprofloxacin (200 mg/l; Bayer Vital), imipenem plus cilastatin $(250 \mathrm{mg} / \mathrm{l}$; MSD) and metronidazole (1 $\mathrm{g} / \mathrm{l}$; Fresenius) were dissolved in $1 \mathrm{~L}$ autoclaved water. Treatment began with an initial per oral challenge of $200 \mu \mathrm{L}$ Abx mixture using an oral gavage. Then Abx mixture was applied via the drinking water ad libitum and continued until the end of the experiment. Drinking water was switched with fresh Abx twice a week. Mice were relocated to new cages every other day to prevent recolonization from feces.

Fecal samples were taken to monitor antibiosis in antibiotic-treated mice. DNA from fecal samples was extracted as described previously (Heimesaat et al., 2010). Briefly, DNA extracts and plasmids were quantified using QuantiT PicoGreen reagent (Invitrogen) and adjusted to $1 \mathrm{ng} / \mu \mathrm{l}$. Main bacterial groups abundant in the murine conventional intestinal microbiota were assessed by quantitative RT-PCR with group-specific 16S rRNA gene primers (Tib MolBiol) (Rausch et al., 2013). The number of 16S rRNA gene copies per ng DNA of each sample was determined and frequencies of respective bacterial groups calculated proportionally to the eubacteria amplicon.

\subsection{Immunology methods}

\subsubsection{Cell Isolation}

Blood immune cells from mice were collected from the vena cava and prepared as previously described (Biswas et al., 2015). Mice were deeply anaesthetized by isoflurane inhalation (CP Pharma) and intracardially perfused with $60 \mathrm{~mL}$ sterile phosphate-buffered saline (PBS) prior to organ extraction. To isolate bone marrow cells, the femur and tibia were isolated and 
surrounding tissue was removed. The bone ends were removed, the bone marrow cells were washed out with FACS buffer using a syringe and a $26 \square$ G needle and sieved through a 40 $\mu \mathrm{m}$ strainer. Brains were homogenized in a buffer containing HBSS (Gibco), 1M HEPES (pH 7.3, Thermo Fisher) and $45 \%$ glucose before sieving through a $70 \mu \mathrm{m}$ cell strainer. The homogenate was fractioned on a discontinuous 30-70\% Percoll gradient (GE Healthcare). Immune cells were collected from the $30 / 70 \%$ Percoll interphase, washed in PBS and stained for flow cytometric analysis.

\subsubsection{Flow cytometry}

Flow cytometry stainings were performed as previously described (Düsedau et al., 2019). Single Cell suspensions were incubated with an anti-FcyllI/Il receptor antibody (clone 93, eBioscience) to block unspecific binding and Zombie NIR ${ }^{T M}$ (Biolegend), a fixable viability dye. Thereafter, cells were stained with fluorochrome-conjugated antibodies against cell surface markers: CD45 (30-F11), CD11b (M1/70), Ly6C (HK1.4), CD40 (HM-40-3), F4/80 (BM8), Ly6G (1A8) and MHCII (M5/114.15.2) all purchased from BioLegend; CD40 (HM-403) purchased from eBioscience in FACS buffer at $4 \square \square^{\circ} \mathrm{C}$ for $30 \square \mathrm{min}$. Matched FMO controls were used to assess the level of background fluorescence in the respective detection channel.

To measure cytokine production, intracellular staining was performed as previously described (Düsedau et al., 2019). Cells were incubated with an anti-FcyllI/Il receptor antibody and Zombie NIRTM (Biolegend). Then surface staining was performed on the cells with antibodies against CD45 (30-F11), CD11b (M1/70), Ly6C (HK1.4) and Ly6G (1A8) in FACS buffer. Cells were then fixed in $4 \%$ paraformaldehyde and permeabilized using Permeabilization buffer (Biolegend). Intracellular proteins were stained with antibodies against TNF (MP6-XT22) and IL-6 (MP5-20F3) purchased from eBioscience. Matched isotype controls were used to assess the level of non-specific binding. Flow cytometric analysis was performed on an Attune NxT Flow Cytometer (Thermo Fisher) and analyzed with FlowJo (version 10, FlowJo LLC). 


\subsubsection{RNA Isolation}

To isolate total RNA, samples were homogenized in BashingBeads tubes (Zymo Research) and RNA was isolated using peqGOLD total RNA kit (Peqlab) according to the manufacturer's instructions.

\subsubsection{RT-qPCR}

Gene expression was determined using the TaqMan® RNA-to-CTTM 1-Step Kit (Life Technologies), as previously described (French et al., 2019; Lang et al., 2018). TaqMan® Gene Expression Assays (Life Technologies) were used for mRNA amplification of Tnf (Mm00443258_m1), II6 (Mm00446190_m1), Ifny (Mm00801778_m1), Gfap (Mm01253033_m1), Cx3cr1 (Mm02620111_s1), Slc17a7 (Mm00812886_m1), Slc1a2 (Mm01275814_m1), Gabra1 (Mm00439046_m1), Gria1 (Mm00433753_m1), Gria2 (Mm00442822_m1), Bdnf (Mm04230607_s1), Ngf (Mm00443039_m1), Ntf3 (Mm01182924_m1), Nox4 (Mm00479246_m1), Cox2 (Mm00478374_m1) and Nos2 (Mm00440485_m1). Expression of Hprt (Mm01545399_m1) was chosen as reference, and target/reference ratios were calculated with the LightCycler® 96 software version 1.1 (Roche). All results were further normalized to the mean of the (naive) group.

\subsection{Slice Electrophysiology}

Slice electrophysiology was performed as described previously (Caliskan et al., 2015; Çalışkan et al., 2019). After 6 weeks of chronic antibiotic treatment, decapitation of mice and brain extraction were performed under deep isoflurane anesthesia. $\sim 400 \mu \mathrm{m}$ thick brain slices that contain ventral-to-mid hippocampus were cut in horizontal plane using an angled platform ( $12^{\circ}$ in the fronto-occipital direction) in ice-cold, carbogenated $\left(5 \% \mathrm{CO}_{2} / 95 \% \mathrm{O}_{2}\right)$ artificial cerebrospinal fluid (aCSF) containing (in $\mathrm{mM}$ ) $129 \mathrm{NaCl}, 21 \mathrm{NaHCO}_{3}, 3 \mathrm{KCl}, 1.6$ $\mathrm{CaCl}_{2}, 1.8 \mathrm{MgCl}, 1.25 \mathrm{NaH}_{2} \mathrm{PO}_{4}$ and 10 glucose (pH 7.4, 300 mosmol / kg) with a vibrating microtome (Campden Instruments; Model 752) and quickly transferred to an interface chamber perfused with aCSF at $32 \pm 0.5^{\circ} \mathrm{C}$ (flow rate: $2.0 \pm 0.2 \mathrm{~mL} / \mathrm{min}$ ). A minimum of one hour of slice recovery was allowed before starting recordings. Field potentials (FP) were recorded using borosilicate glass electrodes filled with aCSF with a resistance of $\sim 1 \mathrm{M} \Omega$. FP responses were evoked with a constant current stimulator and a bipolar tungsten wire stimulation electrode (exposed tips: $\sim 20 \mu \mathrm{m}$; tip separations of $\sim 75 \mu \mathrm{m}$; electrode resistance in aCSF: $\sim 0.1 \mathrm{MOhm}$ ). FP signals were pre-amplified using a custom-made amplifier and low-pass filtered at $3 \mathrm{kHz}$. Signals were sampled at a frequency of $10 \mathrm{kHz}$ and stored on a computer hard disc for off-line analysis (Cambridge Electronic Design, Cambridge, UK). 


\subsubsection{Evoked field potential recordings}

To obtain field excitatory postsynaptic potential (fEPSP) responses from CA (Cornu Ammonis) 3-to-CA1 synapse, the recording electrode was placed at the apical dendrites of area CA1 (Stratum Radiatum: SR) and the bipolar stimulation electrode was placed on the Schaffer collaterals (SC) at the proximal CA1 close to the CA2 subregion. After placing the electrodes, responses were recorded for ten-to-twenty min until they were stabilized (interstimulus interval of $30 \mathrm{sec}$ and stimulation duration of $100 \mu \mathrm{s})$. Then, an input-output (I-O) curve was recorded using intensities ranging from 10 to $50 \mu \mathrm{A}$. This was followed by pairedpulse (PP) recording protocol with intervals ranging from 10 to $500 \mathrm{~ms}$. After PP protocol, baseline responses were recorded for another $20 \mathrm{~min}$ and LTP induction was commenced with a train of 100 pulses $(100 \mathrm{~Hz})$ repeated 2 times with $20 \mathrm{~s}$ interval. This was followed by test pulses recorded for $40 \min (0.033 \mathrm{~Hz})$. MATLAB-based analysis tools were used for the analysis of fEPSPs (MathWorks, Natick, MA). For calculation of fEPSP slopes, the slope $(\mathrm{V} / \mathrm{s})$ between the 20 and $80 \%$ of the fEPSP amplitudes were measured. We also calculated an average baseline transmission rate per slice by dividing each fEPSP slope value with the corresponding FV value followed by averaging these values leading to one transmission rate $\left(\mathrm{ms}^{-1}\right)$ value per slice. Paired-pulse responses were analyzed by dividing the slope of the second fEPSP to the first one. For the analysis of LTP, the data were normalized to baseline responses obtained for 20 min before LTP induction.

\subsubsection{Cholinergic gamma oscillations}

To induce gamma oscillations, the temperature of interface chamber was set to $35^{\circ} \mathrm{C}$ and freshly-diluted carbachol (CBh, $5 \mu \mathrm{M})$ was applied via continuous bath perfusion. Fifty-toseventy min after CBh perfusion, three-to-five min recordings were obtained from pyramidal layer of CA3 subregion (stratum pyramidale: SP). Custom-made spike2 scripts were used to analyze gamma oscillations (Cambridge Electronic Design, Cambridge, UK). From each recording $2 \mathrm{~min}$ artifact free data was extracted, and power spectra were generated using Fast Fourier Transformation with a frequency resolution of $0.8192 \mathrm{~Hz}$. Peak frequency $(\mathrm{Hz})$ and Integrated power $\left(20-80 \mathrm{~Hz} ; \mu \mathrm{V}^{2}\right)$ were calculated from the power spectra. Gamma recordings with peak powers lower than $40 \mu \mathrm{V}^{2}$ and peak frequencies lower than $20 \mathrm{~Hz}$ were discarded. For the autocorrelation analysis auto-correlograms were calculated from the 2 min data. The value of the 2 nd positive peak of auto-correlogram was used to report the gamma correlation strength of local CA3 gamma oscillations. 


\subsubsection{Sharp Wave-Ripples}

Glass electrodes were placed at the SP of CA1 subregion. Data were recorded for three-tofive min and two min artifact free data were extracted as MATLAB files to be further analyzed using a custom written MATLAB-scripts (MathWorks, Natick, MA). For sharp wave (SW) detection, the data was low-pass filtered at $45 \mathrm{~Hz}$ (FFT filter). The SW events were detected with threshold set to 2.5 times the standard deviation (SD) of the lowpass-filtered signal. The minimum interval between two subsequent SW was set to $80 \mathrm{~ms}$. Data stretches of $125 \mathrm{~ms}$ centered to the maximum of the sharp wave event were stored for further analysis. The start and the end point of SW was determined by the points crossing the mean of the data. The area under curve (AUC) was calculated using the low pass filtered data using these two points as the start and end of the SW event. Ripples were isolated using band-pass filter at $120-300 \mathrm{~Hz}$ (FFT filter). $15 \mathrm{~ms}$ before and $10 \mathrm{~ms}$ after the maximum of SW event (25 ms) were stored for further analysis of the ripples. The ripple events were detected with threshold set to 3 times the SD of the bandpass-filtered signal. Ripple amplitude was analyzed using triple-point-minimax-determination. Only ripples with lower than $75 \%$ difference between falling and rising component were included in the analysis. The time between the through of subsequent ripples were used for calculation of ripple frequencies.

\subsection{Statistical analysis}

Electrophysiological data were statistically analyzed using SigmaPlot for Windows Version 11.0 (Systat software). Before statistical comparison of different groups, normality test (Shapiro-Wilk Test) and equal variance test were performed. I-O and PP curves were statistically compared using two-way repeated measures ANOVA. For comparison of baseline transmission rate per slice Student's two-tailed t-test was used. For LTP data, normalized data obtained 30-40 min after LTP induction was used for statistical comparison with Mann-Whitney $U$ test. For hippocampal network oscillations, statistical differences were determined by Student's two-tailed t-test or Mann-Whitney $U$ test. For the statistical comparison of gamma power, log transformed data was used. Data from RT-qPCR, flow cytometry cell populations and cell activation were analyzed by Mann-Whitney $U$ test. All Mann-Whitney $U$ tests were two tailed. Probability values of $p<0.05$ were considered as statistically significant. Sample sizes are provided in figure captions ( $N$ : Number of mice; $n$ : Number of slices). 


\section{Results}

\subsection{Microbiota depletion upon antibiotic treatment}

Adult mice were treated with a broad-spectrum antibiotic cocktail for 4 weeks. This antibiotic cocktail has been shown to effectively deplete the intestinal microbiota (Möhle et al., 2016b). To confirm the eradication of the commensal gut bacterial species, the feces from mice with and without antibiotic treatment (+Abx vs. naïve, respectively) were collected and analyzed by $16 \mathrm{~S}$ rRNA sequencing. As expected, broad-spectrum antibiotic treatment led to an elimination of the most abundant gut bacterial groups, genera and species (Fig. 1). Of note, all fecal samples derived from gut microbiota of Abx-treated mice were culture-negative for aerobic, microaerophilic and obligate anaerobic bacteria applying both, solid and enrichment media (data not shown).

EB

EC

LB

BB

\section{BP}

MIB CC

CL

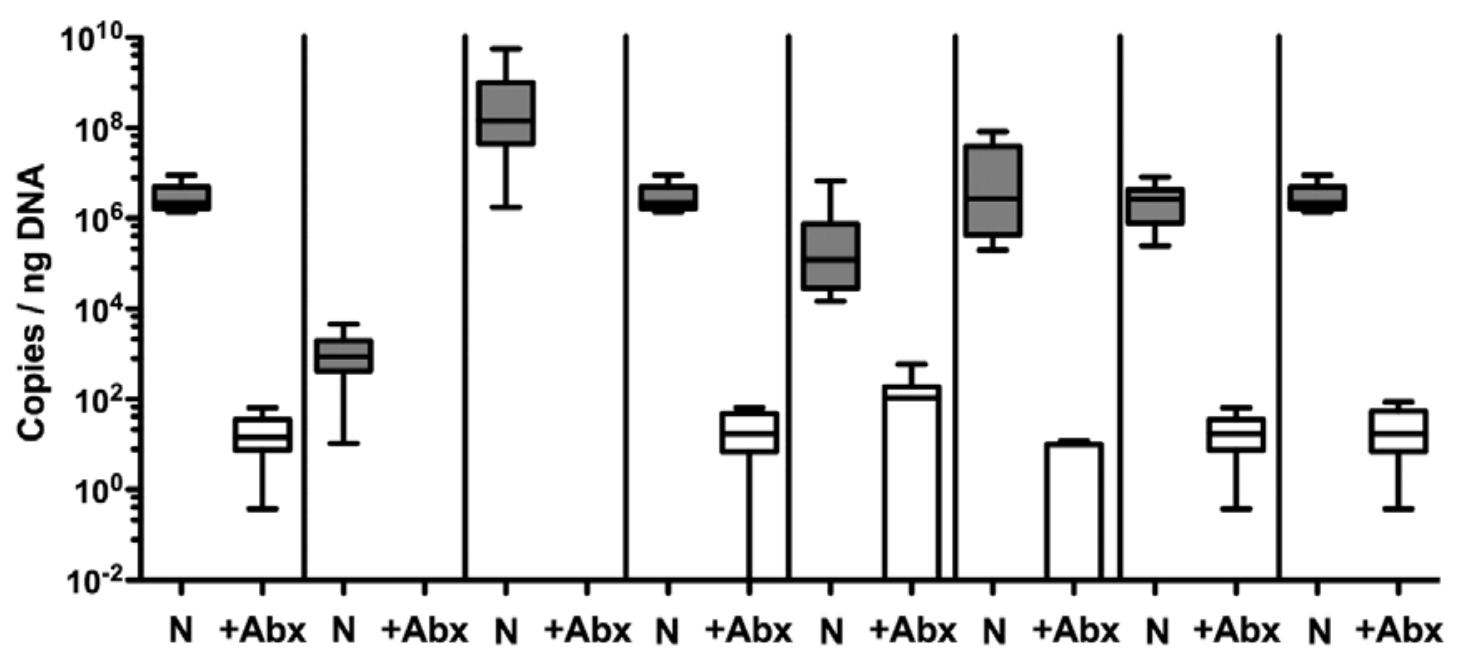

Fig. 1. Microbiota depletion upon antibiotic treatment.

The gut microbiota composition was quantitatively assessed in fecal samples derived from mice that had been subjected to broad-spectrum antibiotic treatment (+Abx; white boxes, $n=10$ ) and from untreated naïve counterparts ( $N$; grey boxes $n=10$ ) applying quantitative $16 S$ rRNA-based bacterial real-time $P C R$ amplifying variable regions of the following bacterial groups (expressed as copies per ng $D N A): E B$, enterobacteria; $E C$, enterococci; $L B$, lactobacilli; $B B$, bifidobacteria; $B P$, Bacteroides/Prevotella species; MIB, Mouse Intestinal Bacteroides; CC, Clostridium coccoides group; $C L$, Clostridium leptum group. Box plots represent the $75^{\text {th }}$ and $25^{\text {th }}$ percentiles of the medians (black bar within box). 
280

281

282

283

284

285

286

287

288

289

290

291

292

293

294

295

296

297

298

299

300

301

302

303

304

305

306

307

308

309

310

311

312

313

\subsection{Reduced frequency of circulating $L y 6 C^{\text {hi }}$ monocytes and increased immune activation upon antibiotic treatment}

Circulating Ly6 $\mathrm{C}^{\text {hi }}$ monocytes exhibit a multifaceted set of functions for the host that have been shown to be beneficial, such as transforming into and replenishing tissue resident DCs or macrophages, or detrimental by exacerbating immunopathology (Cryan and Dinan, 2015; Möhle et al., 2016b; Shi and Pamer, 2011). Thus, we aimed to examine the effect of broadspectrum Abx treatment on the pool of Ly6 $\mathrm{C}^{\text {hi }}$ monocytes in bone marrow, blood and brain by flow cytometry. Monocytes were distinguished from neutrophils via the surface markers CD11b, Ly6C and Ly6G and from microglia by expression of CD45. Monocytes were gated as CD45 ${ }^{\text {hi }}$ CD11 b ${ }^{+}$Ly6C ${ }^{+}$Ly6G- (Fig. 2A, 2C, 2G). Gating of immune cell subsets in the brain that distinguishes recruited myeloid cells from microglia is shown in Fig. $3 \mathrm{C}$. We observed that $\mathrm{Abx}$ treatment led to a significant reduction in the frequency of Ly6C $\mathrm{C}^{\text {hi }}$ cells in bone marrow (Fig. 2B; Mann-Whitney $U$ test; $p=0.0079$ ), circulating in the blood (Fig. 2D; MannWhitney $U$ test; $p=0.008$ ) and those infiltrating the brain (Fig. $2 \mathrm{H}$; Mann-Whitney $U$ test; $\mathrm{p}=0.0079$ ). To investigate if Abx treatment was altering the activation status of the circulating monocytes, we analyzed their expression of MHC II and CD40, markers associated with classically activated M1 immune cells and upregulated upon TLR engagement (Andrade et al., 2005; Sica and Mantovani, 2012). We detected a significant increase in the expression of both MHC II and CD40 in blood Ly6C hi monocytes (Fig 2E-F; Mann-Whitney U test; MHC II, $\mathrm{p}=0.016$; CD40, $\mathrm{p}=0.016$ ) and increase of CD40 on those infiltrating the brain (Fig 2I-J; Mann-Whitney $U$ test; MHC II, $\mathrm{p}=0.52$; CD40, $\mathrm{p}=0.021$ ) upon Abx treatment. These results highlight that prolonged $\mathrm{Abx}$ treatment associates with a global reduction of the available

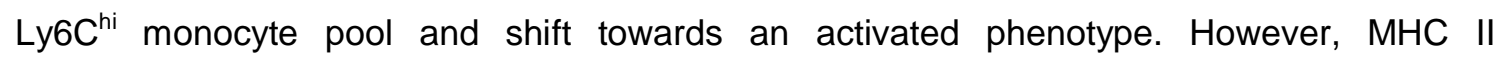
expression remains unaltered when the CNS is infiltrated, suggesting there might be a certain amount of translocation of the remaining gut microbes which promote inflammation locally. We observed an enlarged and inflamed cecum in Abx-treated animals which is comparable to other Abx treatment studies in mice (Ge et al., 2017; Sun et al., 2021). Since the spleen is a primary filter of blood-borne pathogens and antigens and serves as detector of systemic inflammation (Altamura et al., 2001; Cobb et al., 2002), we analyzed the gene expression of TNF and IL-6. Gene expression analysis revealed that Abx treatment led to an increase of both TNF and IL-6 expression compared to naïve animals (Fig 2K; Mann-Whitney $\mathrm{U}$ test; TNF, $\mathrm{p}=0.002 ; \mathrm{IL}-6, \mathrm{p}=0.028)$. These data indicate that microbiota are necessary to maintain the steady-state myeloid populations. 

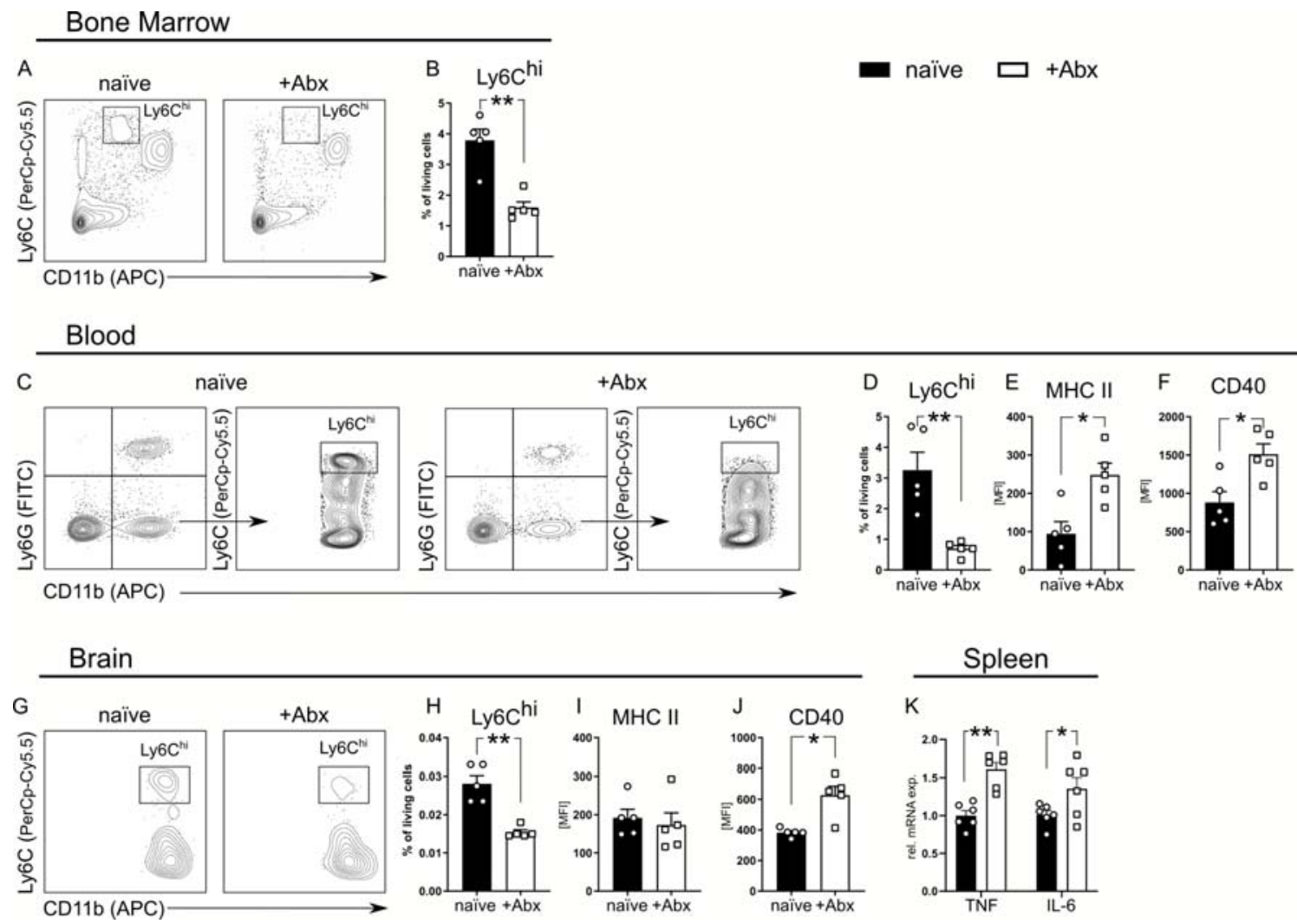

Fig. 2. Reduced frequency of $L y 6 C^{h i}$ monocytes and increased immune activation upon antibiotic treatment

Immune cells from bone marrow, blood and brain were isolated from naïve ( $N=5$ mice) and $A b x$ treated ( $+A b x ; N=5$ mice) mice and analyzed by flow cytometry. $(A, C, G)$ Representative Ly6C $C^{\text {hi }}$ monocyte gating strategies for bone marrow, blood and brain. Events were gated on singlets and cells were excluded based on forward and side scatter (FSC/SSC) and viability staining (dead cells).. Ly6C $C^{\text {hi }}$ monocytes were defined as CD11 b+ Ly6G Ly6C Chi (bone marrow, blood) or CD45+CD11b+Ly6GLy6Chi (brain, to exclude CD45lowCD11b+ microglia). $(B, D, H)$ The frequency of $L y 6 C^{\text {hi }}$ monocytes is presented as the percentage of living single cells. $(E, F, I, J)$ The surface expression of MHC II and CD40 was determined on Ly6Chi monocytes in naïve and Abx-treated mice by flow cytometric analysis and presented as median fluorescence intensity (MFI). Spleens were collected from naïve $(\mathrm{N}=6)$ and $A b x$-treated $(\mathrm{N}=6)$ mice and were homogenized. $(K)$ Relative mRNA levels were normalized to the mean expression of the naive control group. Symbols represent individual animals. Data is representative of three independent experiments presented as mean + SEM.

\subsection{Antibiotic treatment reduces CX3CR1 expression and increases activation of} resident microglia

332 Microglia and recruited myeloid cells are vital for sustaining tissue homeostasis in the CNS 333 (Shechter and Schwartz, 2013). Given the observed reduction of circulating Ly6C ${ }^{\text {hi }}$ monocytes and increased splenic TNF and IL-6 expression, we decided to first assess if Abx 
335

336

337

338

339

340

341

342

343

344

345

346

347

348

349

350

351

352

353

354

355

356

357

358

359

360

treatment was also altering the gene expression of proinflammatory markers TNF, IL-6 and IFNy in whole brain homogenates of naïve and Abx-treated mice. In contrast to the periphery, we detected a decrease of TNF gene expression and no difference in IL-6 or IFNY gene expression (Fig. 3A; Mann-Whitney $U$ test; TNF, $p=0.02$; IL-6, $p=0.35$; IFNy, $p=0.99$ ) in Abx-treated mice. TNF is primarily synthesized by glial cells in the brain (McCoy and Tansey, 2008). To determine if a particular resident glia cell type is altered upon Abx treatment, we measured the gene expression of glial activation markers GFAP (astrocytes) and CX3CR1 (microglia) (Li et al., 2020; Wolf et al., 2013). Following antibiotic treatment, GFAP expression was unchanged whereas expression of CX3CR1 was reduced (Fig. 3B, MannWhitney $U$ test; $C X 3 C R 1, p=0.03$; GFAP, $p=0.8$ ), indicating that microglia in particular, are influenced by Abx treatment.

CX3CL1 is mainly secreted by neurons and induces neuroprotective effects via interaction with its receptor CX3CR1 on microglia (Nash et al., 2015). CX3CL1 thereby downregulates the production of proinflammatory mediators such as TNF, NO and superoxides. Based on the observed reduction in CX3CR1, we next examined the activation status of resident microglia (CD11 $\mathrm{b}^{+} \mathrm{CD} 45^{\text {int }}$ cells) via flow cytometry, using MHC II and CD40 as phenotypic markers (Fig. 3C). Upon Abx treatment, expression of MHC II (Fig. 3D; Mann-Whitney U test; $p=0.028$ ) and CD40 (Fig. 3E; Mann-Whitney $U$ test; $p=0.020$ ) significantly increased. To further investigate the level of microglia activation, cytokine production was assessed. Here, microglia of Abx-treated mice showed a significantly higher IL-6 expression (Fig. 3F; MannWhitney $U$ test; $p=0.026$ ) compared to naïve mice whereas TNF production remained unchanged (Fig 3G; Mann-Whitney $U$ test; $p=0.11$ ). However, there were significantly more microglia producing TNF in Abx-treated mice compared to naïve (Fig. 3H; Mann-Whitney $U$ test; $p=0.028$ ). These data imply that the gut microbiome plays an important role in modulating and maintaining microglial function. 
A

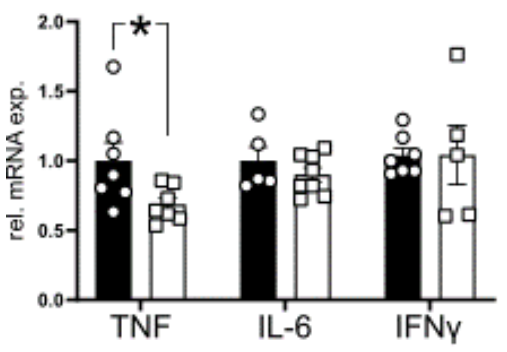

B

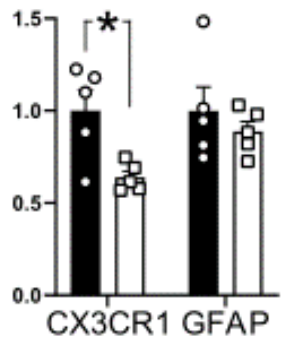

C

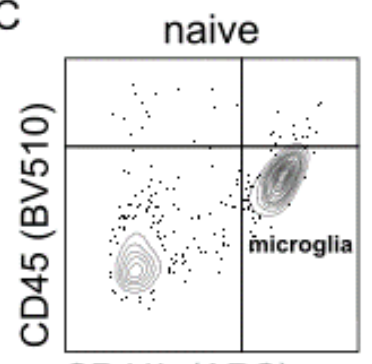

CD11b (APC)
362

363

364

365

366

367

368

369

370

371

372

373

374

375

376

377

378

379

380

381

382

383
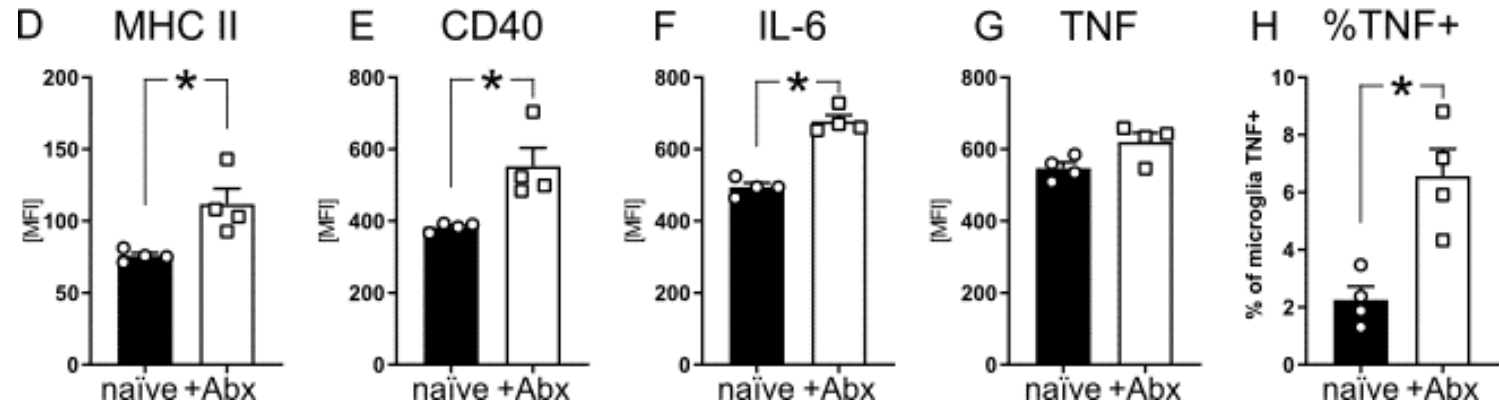

Fig. 3. Increased activation of microglia upon antibiotic treatment

Relative expression of $m R N A$ levels of $(A)$ proinflammatory and $(B)$ glial genes in naïve $(N=5-7)$ and Abx-treated ( $N=5-8)$. Relative mRNA levels were normalized to the mean of the naivve control group. (C-H) Immune cells from brains of naïve $(\mathrm{N}=4)$ and Abx-treated $(\mathrm{N}=4)$ were isolated and analyzed by flow cytometry. (C) Representative gating strategy of brain immune cells. Events were gated on singlets and cells were excluded based on forward and side scatter (FSC/SSC) and viability staining (dead cells). $C D 11 b^{+} C D 45^{\text {int }}$ cells were defined as microglia. The surface expression of (D) MHC II, (E) CD40 and intracellular production of (F) IL-6 and (G) TNF by microglia were quantified and presented as MFI of their respective fluorochrome. $(H)$ The percentage of microglia that are positively producing TNF. RNA was isolated from whole brain homogenate of naïve and Abx-treated $(+A b x)$ mice for RT-qPCR analysis. Symbols represent individual animals. Data is representative of three independent experiments and presented as mean + SEM.

\subsection{Enhanced iNOS expression and reduced NGF upon antibiotic treatment}

Microglia are known to upregulate reactive oxygen species (ROS) and reactive nitrogen species (RNS) when activated (Mander and Brown, 2005; Ta et al., 2019). In excessive quantities, ROS can lead to axonal and neuronal loss, for example, in neurodegenerative diseases (Barbeito et al., 2004; Okuno et al., 2005). To investigate if Abx treatment leads to alterations in the gene expression of RNS or ROS producing enzymes, we measured the gene expression of NADPH-Oxidase (Nox4), cyclooxygenase 2 (Cox2) and inducible nitric oxide synthase (iNOS; Nos2), two superoxide and NO producers affiliated with microglial 
function (Dugan et al., 2009; Ta et al., 2019) via RT-qPCR analysis. Upon Abx treatment, no change was detected in Nox4 or Cox2 gene expression whereas Nos2 expression was significantly increased (Fig. 4A; Mann-Whitney U test; Nox4, $p=0.99$; Cox2, $\mathrm{p}=0.27$; iNOS, $\mathrm{p}=0.0079$ ). Upregulation of iNOS along with microglia activation has been linked to disturbances in fast neuronal network oscillations underlying perception, attention and memory (Ta et al., 2019). Moreover, we previously demonstrated (Möhle et al., 2016b) that the same antibiotic cocktail disrupts learning and memory. Thus, we hypothesized that Abx treatment may lead to dysregulation of crucial neurotrophins, and proteins involved in synaptic homeostasis. To determine if Abx treatment led to changes in expression of neurotrophins, we measured the mRNA levels of brain-derived neurotrophic factor (BDNF), nerve growth factor (NGF) and neurotrophin-3 (NTF3). There were no gene expression changes observed in BDNF or NTF3 between the groups whereas NGF gene expression was diminished in Abx-treated brains (Fig. 4B; Mann-Whitney $U$ test; Bdnf, $\mathrm{p}=0.41$; Ngf, $\mathrm{p}=0.042$, Ntf3, $\mathrm{p}=0.52$ ). To explore whether microglial activation and increased Nos2 expression result in changes to excitatory or inhibitory signal transduction, expression levels of receptors and transporters involved in glutamatergic (Glur1/2, VGLUT1, EAAT2) and GABAergic signaling $\left(G_{A B A_{A}} \alpha 1\right)$ were determined. When looking at the gene expression for all synaptic markers, we observed no change in expression after Abx treatment (Fig. 4C; Mann-Whitney $U$ test; $p>0.8$ for all).

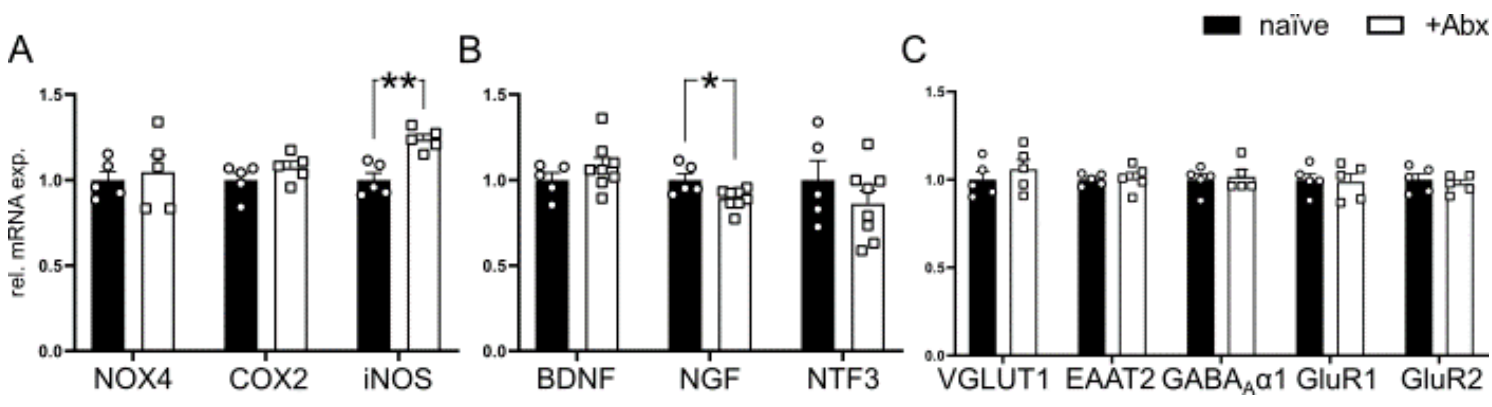

Fig. 4. Increased iNOS and decreased NGF gene expression upon antibiotic treatment

$R N A$ was isolated from whole brain homogenate of naïve and Abx-treated (+Abx) mice for $R T-q P C R$ analysis. Relative expression of $m R N A$ levels of $(A)$ reactive species, $(B)$ neurotrophins and $(C)$ synaptic genes in naïve $(N=5)$ and $+A b x(5-8)$ mice. Relative mRNA levels were normalized to the mean of the naïve control group. Symbols represent individual animals. Data is representative of three independent experiments. Data is presented as mean + SEM.

\subsection{Reduced hippocampal synaptic transmission upon antibiotic treatment}


413 Due to the well-established role of cytokines and neurotrophins in sustaining synaptic

414 transmission and plasticity (Albensi and Mattson, 2000; Beattie et al., 2002; Conner et al., 415 2009; Curran and O'Connor, 2003; Hoshino et al., 2017; Leal et al., 2017), we assessed the 416 changes in synaptic physiology at the hippocampal CA3-to-CA1 synapse upon chronic Abx 417 treatment. We observed a profound decrease in the baseline transmission evident by lower 418 dendritic fEPSP responses to increasing stimulation strengths (Fig. 5A). We detected a 419 strong stimulation intensity $\times$ fEPSP slope interaction (Two-way repeated measures ANOVA; $420 \mathrm{~F}(6,27)=4.569, \mathrm{p}<0.001)$ with significant reduction at $20 \mu \mathrm{A}$ to $50 \mu \mathrm{A}$ stimulus intensities 421 (Fisher's LSD post hoc comparison; $20 \mu \mathrm{A}: \mathrm{p}=0.25 ; 30 \mu \mathrm{A}: \mathrm{p}=0.012 ; 40 \mu \mathrm{A}: \mathrm{p}=0.008 ; 50$ $422 \mu \mathrm{A}: \mathrm{p}=0.008$ ). On the other hand, we observed no Abx treatment effect (Fig. 5B; Two-way 423 repeated measures ANOVA; $F(1,21)=4.569, p<0.001)$ for the presynaptic fiber volley $(F V)$ 424 amplitudes suggesting a potential Abx-induced alteration restricted to the postsynaptic part.

425 Comparison of average baseline transmission rates per slice (see methods), also showed a 426 reduced baseline transmission rate (Fig. 5C; Student's two-tailed t-test; $T(21)=2,156, p=$ 427 0.043) suggesting a reduced efficacy of synaptic transmission at this hippocampal synapse. 428 Assessment of short-term plasticity via paired-pulse protocol revealed no statistical 429 differences after Abx treatment (Fig. 5D; Two-way repeated measures ANOVA; F (1, 27) = $4301.378, p<0.251$ ). Last, we assessed long-term potentiation with a HFS protocol (see 431 methods). Both control and Abx-treated mice showed a strong potentiation which stayed 432 stable up to 40 min after HFS (Fig. 5E). Comparison of normalized fEPSP slope values 30$43340 \mathrm{~min}$ after HFS showed no effect of Abx treatment (Fig. 5F; Mann-Whitney $U$ test; $p=$ 434 0.075). These data suggest that chronic Abx treatment results in a profound decrease in the 435 baseline synaptic transmission at the hippocampal CA3-CA1 synapse without altering 436 synaptic plasticity. 

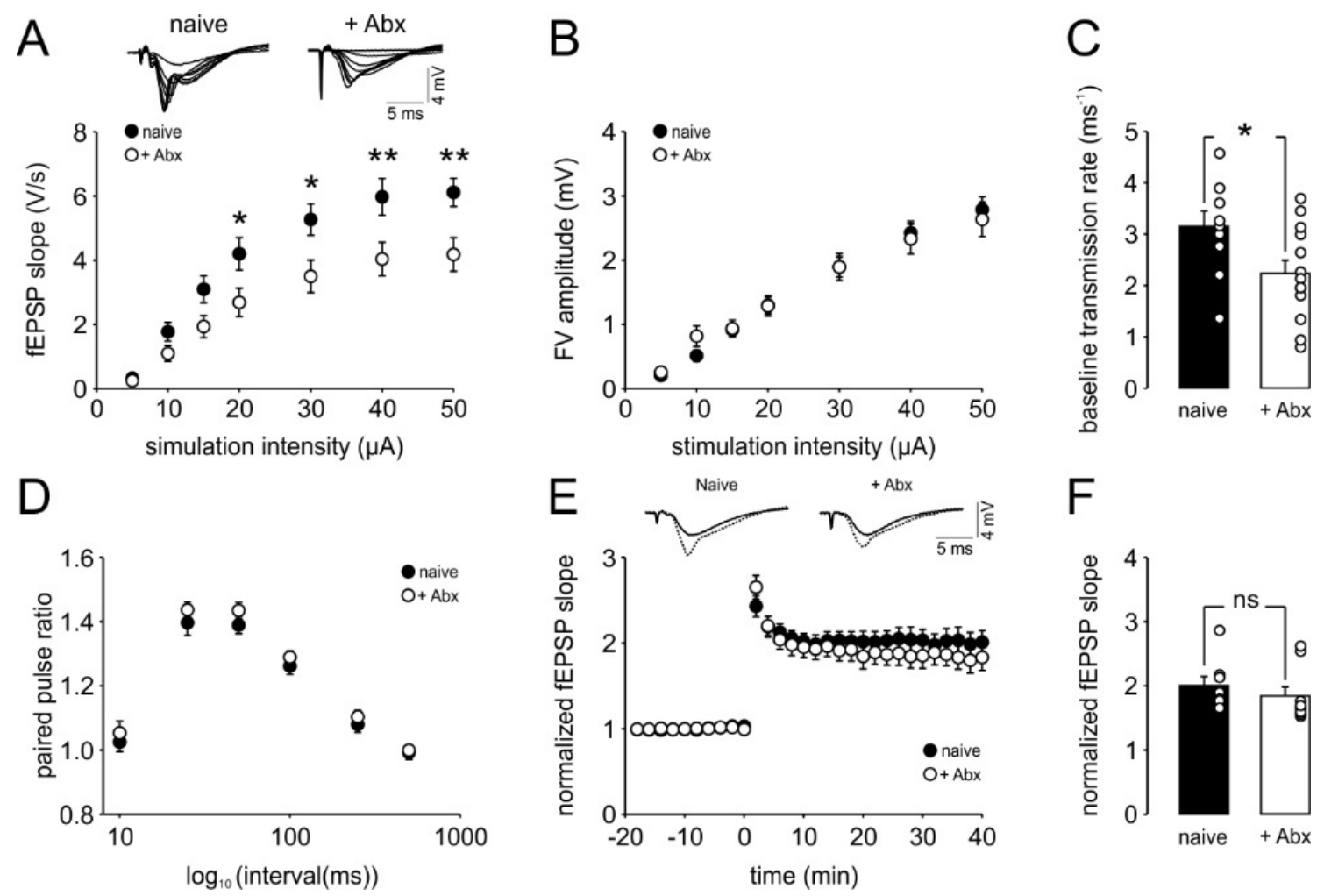

438

439

440

441

442

443

444

445

446

447

448

449

450

451

452

453

454

455

456

457

458

459

Fig 5. Reduced baseline transmission at the Schaffer collateral (SC)-CA1 synapse after chronic antibiotic treatment $(+\boldsymbol{A b x})$. (A) Input-output curve showing reduced CA1 field excitatory postsynaptic potentials (fEPSP) in $+A b x$ mice ( $N=5$ mice, $n=13$ slices) in comparison to naive mice ( $N=3$ mice, $n=10$ slices). (B) Input-output curve showing unaltered presynaptic fiber volley amplitudes in + Abx mice ( $N=5$ mice, $n=19$ slices) in comparison to naive mice ( $N=3$ mice, $n=10$ slices). (C) Summary graph illustrating a reduced baseline transmission rate (averaged fEPSP slope / FV amplitude values per slice) at the SC-CA1 synapse after Abx treatment (Naive mice: $N=3$ mice, $n=10$ slices; + Abx mice: $N=5$ mice, $n=13$ slices). (D) Short-term plasticity is not altered in + Abx mice evident by the unchanged paired pulse ratios (Naive mice: $N=3$ mice, $n=10$ slices; + Abx mice $N=5$ mice, $n=19$ slices). (E) Increase in the $f E P S P$ responses upon high frequency stimulation (HFS) of the $S C$ in the + Abx mice are comparable to the control mice indicating no change in long-term plasticity (LTP). (F) Analysis of normalized fEPSP values obtained during 30-40 min after HFS reveal no significant alteration between the naive ( $N=4$ mice, $n=8$ slices) and $+A b x$ ( $N=5$ mice, $n=9$ slices) mice. Data are presented as mean \pm SEM.

\subsection{Reduced hippocampal gamma oscillations upon antibiotic treatment}

In order to assess the impact of the reduced hippocampal synaptic transmission on behaviorally-relevant activities in the hippocampus, first, we recorded cholinergic gamma oscillations in the CA3 subregion. Gamma oscillations appeared after ten-to-fifteen min and stabilized after 50 min after CBh perfusion (Fig. 6A). Analysis of the power spectra revealed a shift in the peak frequency of gamma oscillations (Fig. 6B-C; Mann-Whitney $U$ test; $p<$ 
460 0.001) and strong reduction in the gamma power (Fig. 6C-D; Student's two-tailed t-test; T(28)

$461=2.591, p=0.015)$. Next, we measured local synchronization of gamma oscillations and 462 found no significant change in the gamma correlation upon Abx treatment (Fig. 6E-F; Mann463 Whitney $U$ test; $p=0.271$ ). Overall, gamma oscillations appear to be reduced in power upon 464 chronic Abx treatment.

465

A

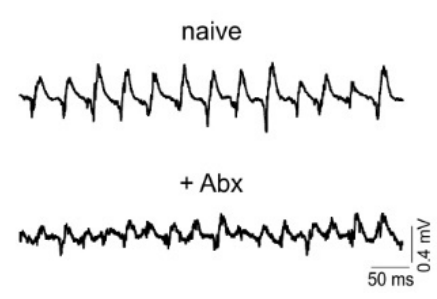

C

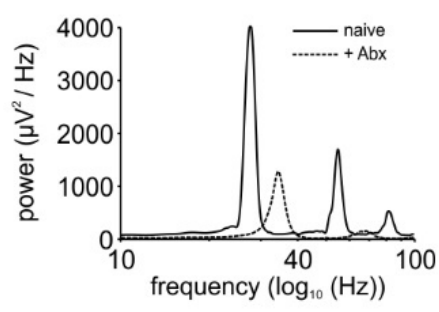

E

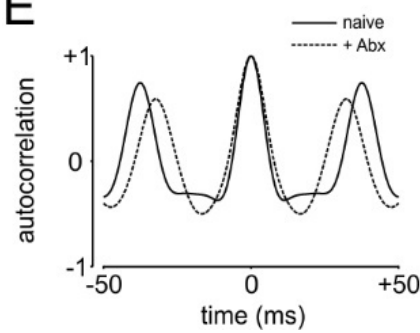

B

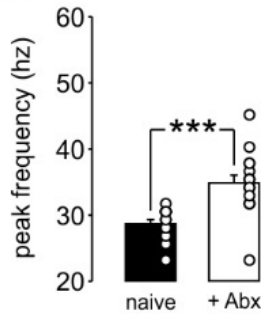

$\mathrm{D}$
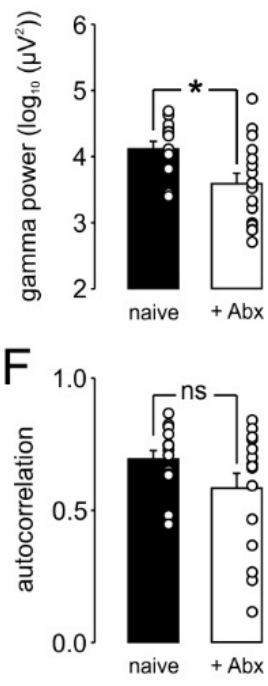

466

Fig 6. Reduced hippocampal cholinergic gamma oscillations after chronic antibiotic treatment.

(A) Representative carbachol (CBh, $5 \mu M$ ) induced local field potential (LFP) traces from hippocampal $C A 3$ of $+A b x$ ( $N=5$ mice, $n=16$ slices for all parameters) and naive ( $N=4$ mice, $n=14$ slices for all parameters) mice. (B) Summary graph illustrating an increased gamma peak frequency in + Abx mice. (C) Representative power spectra illustrating a shift in the main gamma peak frequency and a reduction in gamma-range oscillation power in the hippocampal CA3 of $+A b x$ mice. (D) Summary graph illustrating an increased gamma power $(20-80 \mathrm{~Hz})$ in the hippocampal CA3 of $+A b x$ mice. Representative auto-correlograms of CA3 LFP gamma oscillations. (E) Representative autocorrelograms illustrating a shift in the $2^{\text {nd }}$ positive peak indicating a reduced duration of gamma cycles, thus, increased gamma peak frequency, in $+A b x$ mice (F) Summary graph showing no significant change in the amplitude of the $2^{\text {nd }}$ peak of the gamma auto-correlograms of $+A b x$ mice in comparison to naive mice. Data are presented as mean \pm SEM. 


\subsection{Increased incidence of sharp waves upon antibiotic treatment}

482

Horizontal or transverse-like slices obtained from the ventral-to-mid portion of the hippocampus generates spontaneous sharp wave-ripple (SW-R) activity (Caliskan et al., 2015; Çalışkan et al., 2016; Maier et al., 2003). Both in vivo and in vitro, these events have been associated with emotional and spatial memory consolidation (Çalışkan et al., 2016; Girardeau et al., 2017, 2009). Thus, we assessed the effect of chronic Abx treatment on spontaneous SW-R activity in the CA1 subregion (Fig. 7A-B). We found a specific increase in the incidence of SW (Fig. 7C; Student's two-tailed t-test; T(40) $=-3.291, p=0.002$ ) without any alterations in the SW area (Fig. 7D; Mann-Whitney $U$ test; $p=0.712$ ), ripple amplitude

A naive

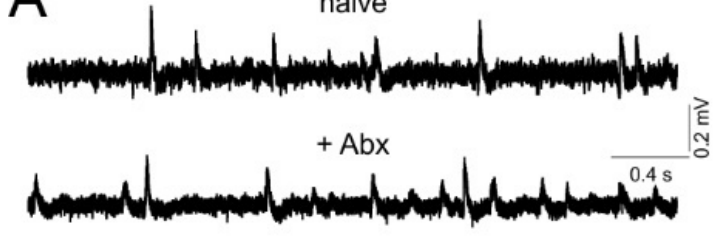

C
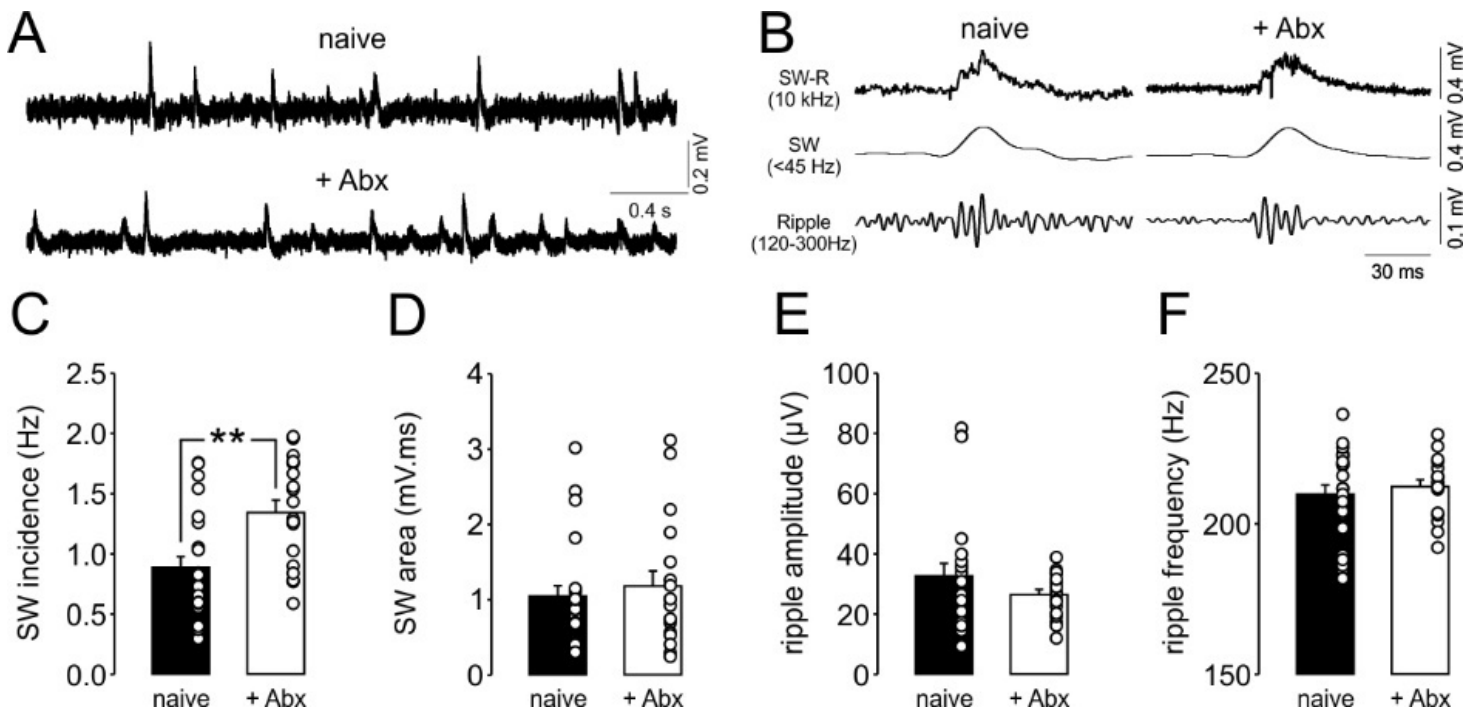

Fig 7. Increased incidence of sharp wave-ripples in the CA1 subregion of the hippocampus after chronic antibiotic treatment. (A) Representative LFP traces showing an increased number of sharp wave-ripples $(S W-R)$ in the $C A 1$ of $+A b x(N=5$ mice, $n=18$ slices for all parameters) in comparison to $-A b x$ mice ( $N=7$ mice, $n=24$ slices for all parameters). (B) Representative LFP traces of single $S W$-R, low-pass filtered SW $(<45 \mathrm{~Hz})$ and band-pass filtered ripples (120-300 Hz). Summary graphs showing (C) an increased CA1 SW incidence and no change in (D) SW area, (E) ripple 
503

504

505

506

507

508

509

510

511

512

513

514

515

516

517

518

519

520

521

522

523

524

525

526

527

528

529

530

531

532

533

534

535

536

537

538

\section{Discussion}

In the current study, we demonstrate that alterations in homeostatic immunoregulation induced by gut dysbiosis is associated with impaired synaptic transmission and behaviorallyrelevant brain rhythms in the hippocampus. We show that a four-week antibiotic treatment leads to a virtual depletion of the gut microbiota and substantial reduction in the circulating ${\text { Ly } 6 \mathrm{C}^{\text {hi }}}$ monocyte pool and decreased infiltration into the central nervous system (CNS). The reduced recruitment of peripheral cells was accompanied by increased activation of resident microglia with increased iNOS expression and reduced levels of the neurotrophin NGF and TNF. In line with the role of cytokines and neurotrophins in the maintenance of synaptic transmission, we observed a profound reduction of hippocampal CA3-to-CA1 synaptic transmission and cholinergic gamma oscillations. Thus, our study provides further insights into the interaction between gut microbiota and the CNS which is mediated by the immune system.

We have previously highlighted the role of circulating Ly6C $\mathrm{C}^{\text {hi }}$ monocytes in gut-brain communication and their contribution to the recovery of Abx-induced impairment of hippocampal neurogenesis and cognitive function in mice (Möhle et al., 2016b). In the present study, we further investigated the consequences of the alterations in the circulating ${\text { Ly } 6 C^{\text {hi }}}$ monocyte pool upon Abx-induced dysbiosis of the gut microbiota. We provide evidence for a shift towards an activated state of Ly6 $\mathrm{C}^{\text {hi }}$ monocyte via enhanced expression of MHC II and CD40 (Fig. 2). A possible explanation for this observation could be an enhanced commensal translocation across intestinal epithelium and subsequent promotion of inflammation as we did observe enhanced proinflammatory gene expression of TNF and IL-6. This aligns with previous studies that described increased IL-17 and IFNy after Abxinduced bacterial translocation or enhanced IL-6 and TNF in response to local bacterial infection in the spleen (Knoop et al., 2016; Straub et al., 2000).

Peripheral inflammation is associated with altered proinflammatory cytokine levels and circuit excitability in the brain (Galic et al., 2012). To gain insights into the potential regulation of proinflammatory cytokines in the CNS upon Abx treatment, we assessed the expression levels of TNF, IL-6 and IFNY in whole brain homogenates (Fig. 3). Surprisingly, we found a moderate reduction in the TNF expression. In the brain, TNF is primarily synthesized in nonneuronal cell populations, including microglia and astrocytes (McCoy and Tansey, 2008). In contrast to the reduced TNF expression, we found a reduction in microglia-specific CX3CR1 expression, whereas we did not observe an altered GFAP expression (Fig. 4). Furthermore, we detected increases in markers that would suggest an activated microglial phenotype. Given the global reduction of TNF in the brain and the only basal infiltration of peripheral immune cells, it is likely that the observed increase in the percentage of TNF-producing 
539 microglia has only minimal influence on higher brain functions. While TNF has 540 neuromodulatory effects by influencing neurotrophin production at low concentrations (Perry 541 et al., 2002), high TNF concentrations are linked to neurotoxicity (Probert, 2015). Thus, 542 prolonged Abx treatment could presumably increase TNF levels and result in detrimental 543 effects. In line with the concept, a reduction in CX3CR1 expression or reduced interaction 544 with its ligand CX3CL1 is associated with increased microglia activation and overproduction 545 of pro-inflammatory cytokines (Cardona et al., 2006; Lyons et al., 2009; Rogers et al., 2011). 546 Moreover, $\mathrm{CX} 3 \mathrm{CR} 1^{-1}$ mice show deficits in hippocampal synaptic physiology and reduced 547 coherence of distinct LFP network activities, including gamma oscillations (Rogers et al., 548 2011; Zhan et al., 2014). These observations are consistent with the involvement of activated 549 microglia as mediators of reduced hippocampal synaptic transmission and gamma oscillatory 550 activity after prolonged Abx treatment.

551 Reduced glial TNF production and subsequent signaling in neurons leads to impairment of 552 excitatory synaptic transmission and synaptic scaling via regulation of surface AMPA-R 553 levels (Beattie et al., 2002; Stellwagen and Malenka, 2006). In our experiments, Abx 554 treatment lead to reduced expression of TNF and reduced hippocampal CA1 synaptic 555 strength upon (Fig. 5), known to be dependent on intact AMPA-R function (Chater and Goda, 556 2014). However, transcriptional alterations of two main AMPAR subunits (GluR1, GluR2), the 557 astrocytic (EAAT2), vesicular glutamate transporter (VGLUT1) and inhibitory GABA receptor $558\left(\mathrm{GABA}_{A} \alpha 1\right)$ which play an important role in sustaining synaptic transmission and plasticity in 559 the brain, were not detected (Fig. 4). Thus, future studies assessing the cell-surface 560 expression levels of AMPA-R in a brain-region specific manner might help in gaining more 561 mechanistic insights into the impact of Abx treatment on circuit excitability.

562 Microglia activation can lead to increased generation of reactive oxygen species (ROS) and 563 reactive nitrogen species (RNS) (Ramalingam and Kim, 2012). Their imbalanced production 564 is associated with excessive oxidative stress which can be detrimental to cellular function 565 and is commonly observed during many pathological conditions (Dröge and Schipper, 2007; 566 Okuno et al., 2005; Sesti et al., 2010). Of note, cholinergic gamma oscillations appear to 567 require high levels of mitochondrial activity and use high levels of oxidative capacity (Kann et 568 al., 2011). These features make gamma oscillations especially vulnerable to oxidative stress 569 (Hasam-Henderson et al., 2018). While gene expression levels of ROS producing enzymes 570 NADPH-oxidase (Nox4) or Cyclooxygenase 2 (Cox2) were not altered, we measured a 571 significant increase in the expression of iNOS (Nos2) (Fig. 4). This finding is particularly 572 interesting in the light of the recent reports demonstrating that iNOS-mediated NO release 573 can modulate cholinergic hippocampal gamma oscillations in vitro (Papageorgiou et al., 574 2016; Ta et al., 2019) and open a new perspective for synergistic interactions between 
575 microglia/microglia-associated factors and sustainment of gamma oscillations in the context 576 of disease pathology (Adaikkan and Tsai, 2020; laccarino et al., 2016; Martorell et al., 2019).

577 Future studies investigating specific microglial factors and their relation to intrinsic oscillatory 578 brain activities in a brain-region specific manner will further help to understand the impact of 579 long-term Abx treatment on distinct brain functions.

580 Gut dysbiosis can have major impacts on CNS function that results in altered cognitive 581 function, mood and behavior (Irwin and Miller, 2007; Mayer, 2011; Sarkar et al., 2018). 582 Converging evidence from our previous studies and by other groups indicate that Abx 583 treatment leads to a distinct alteration memory, as evidenced by the novel object recognition 584 test (Desbonnet et al., 2015; Fröhlich et al., 2016b; Möhle et al., 2016b; Sarkar et al., 2020). 585 In addition to the previously described reduced hippocampal neurogenesis as a correlate of 586 this memory deficit (Möhle et al., 2016b), now, we found a strong reduction in CBh-induced 587 cholinergic gamma oscillations in the hippocampus upon Abx treatment (Fig. 6). In 588 accordance, increase in CA3 gamma power and gamma coherence along the hippocampal 589 CA3-CA1 axis appears to be critical for novel object recognition/exploration (Trimper et al., 590 2014). Furthermore, deficit in object place recognition task in an Alzheimer's mouse model 591 can be rescued by optogenetic gamma stimulation (Etter et al., 2019). Thus, an insufficient 592 CA3 gamma power might underlie the deficit in this hippocampus-dependent memory. In 593 support of this argument, we describe a mild but significant reduction in, which is known to 594 positively regulate the cholinergic activity in the hippocampus (Conner et al., 2009). 595 Furthermore, a recent study (Sarkar et al., 2020) provides evidence for a substantial 596 elevation in brain ACh esterase levels upon Abx treatment. Such an increase in the ACh esterase might reduce extracellular ACh concentration and potentially diminish cholinergic gamma oscillations (Hollnagel et al., 2015). Interestingly, chronic Abx treatment leads to an 599 aberrant sleep/awake architecture and a mild reduction in theta power during sleep 600 suggesting a potential reduction of co-occurring gamma oscillations in vivo (Ogawa et al., 601 2020). Thus, we propose that, upon Abx treatment, a potential decline in the cholinergic 602 tonus associated with reduced levels of NGF might contribute to the impairment of 603 cholinergic gamma oscillations.

604 The duration of Abx-administration appears to be critical for determining the impact of gut 605 dysbiosis on emotional behavior. While relatively short periods of Abx treatment $(\sim 1 \mathrm{w}) \mathrm{had}$ 606 no apparent effects on innate anxiety (Fröhlich et al., 2016b), longer Abx treatment ( 7 w) 607 (Desbonnet et al., 2015) from weaning on reduced anxiety-like behavior in mice. Similarly, 608 germ-free mice show also reduced anxiety-like behavior (Heijtz et al., 2011; Neufeld et al., 609 2011) resembling the behavioral alterations observed with longer periods of Abx treatment. 610 Our electrophysiological recordings were obtained from slices obtained from the ventral-to- 
611 mid hippocampus. The ventral sector of the hippocampus has been implicated in mediating 612 anxiety (Fanselow and Dong, 2010) and its lesion leads to reduced innate anxiety (Kjelstrup 613 et al., 2002). Thus, a reduced baseline transmission and diminished gamma oscillations in 614 the ventral hippocampus align well with a reduced anxiety and might be involved in the 615 anxiolytic effects reported for long-term Abx treatment.

616 In addition to gamma oscillations, sharp wave-ripples (SW-R) are also generated in the CA3617 to-CA1 axis of the hippocampus and the balance between these network activities is highly 618 dependent on cholinergic tonus (Buzsáki, 2015; Çalışkan et al., 2016; Çalışkan and Stork, 619 2019). We found a profound increase in the incidence of SW-R upon long-term Abx 620 treatment (Fig. 7), similar to the findings of a previous study demonstrating an increased 621 hippocampal SW incidence in association with retarded extinction of contextual fear 622 memories (Çalişkan et al., 2016). Accordingly, a recent work (Chu et al., 2019) shows that 623 Abx-treated mice show impaired extinction of a cued fear memory. Both of these fear 624 paradigms are dependent on the functional interactions between the hippocampus and 625 amygdala (Çalışkan et al., 2019) and amygdalar activity appears to be recruited during SW 626 events (Girardeau et al., 2017). Thus, increased SW incidence might partially predispose for 627 re-consolidation of original fear memories and impair their extinction in Abx-treated mice. To the best of our knowledge, this is the first study demonstrating the alterations in the LFP 629 hippocampal network oscillations and synaptic properties of the hippocampus after long-term 630 Abx treatment in mice. These electrophysiological alterations are associated with an 631 enhanced activation of peripheral immune cells and microglia as well as alterations in the 632 expression of neurotrophic factors and cytokines in the brain. A very recent study on mice 633 raised under GF conditions from birth onwards, has demonstrated a reduced LTP in the 634 hippocampal CA1 of male GF mice while no alteration was detected in the baseline synaptic 635 transmission (Darch et al., 2021). As we found a profound reduction in the baseline synaptic 636 transmission but only a tendency for a reduction in the CA1 LTP (Fig. 5), a functional 637 microbiome during the early stages of life appears to be important for the sustainment of 638 hippocampal plasticity during adulthood. Thus, studies targeting distinct developmental 639 stages and adulthood are needed to elucidate the impact of Abx treatment on synaptic and 640 cognitive function in an age-dependent manner. Further studies that assess more defined 641 brain areas using causal intervention methods will increase our understanding of the brain642 region specific impact of Abx treatment. The observed electrophysiological and gene 643 expression changes after depletion of gut flora may have broader implications when 644 considering psychiatric and neurodegenerative conditions associated with specific alterations 645 in the immune homeostasis in humans. 


\section{Declaration of Conflict of interest}

647 The authors declare that they have no known competing financial interests or personal 648 relationships that could have appeared to influence the work reported in this paper.

649

\section{Acknowledgements}

651 We are grateful to A. Koffi von Hoff, F. Blitz, G. Reifenberger, D. Zabler and S. Stork for 652 excellent technical assistance and to A. Bohnstedt and D. Al-Chackmakchie for excellent 653 animal care. We are grateful to Dr. J.O. Hollnagel for supporting us with the MATLAB-based 654 analysis tools.

655

\section{Funding}

657 The work was supported by grants from the German Research Foundation (362321501/RTG 6582413 SynAGE to IRD and OS, and CRC1436/A07 to OS) and the Center for Behavioural 659 Brain Sciences - CBBS promoted by Europäische Fonds für regionale Entwicklung - EFRE 660 (ZS/2016/04/78113) to GC and CBBS - ScienceCampus funded by the Leibniz Association 661 (SAS-2015-LIN-LWC) to GC and OS. This work was also supported by the European 662 Structural and Investment Funds (ESF, 2014-2020; project number ZS/2016/08/80645) to 663 IRD. MMH received grant support from the German Federal Ministries of Education and 664 Research (BMBF; zoonoses research consortium PAC-Campylobacter, IP7 / 01KI1725D) 665 and from the Federal Ministry for Economic Affairs and Energy (ZIM, ZF4117908 AJ8). 


\section{References}

Adaikkan, C., Tsai, L.H., 2020. Gamma Entrainment: Impact on Neurocircuits, Glia, and Therapeutic Opportunities. Trends Neurosci. 43, 24-41. https://doi.org/10.1016/j.tins.2019.11.001

Albensi, B.C., Mattson, M.P., 2000. Evidence for the involvement of TNF and NF-kB in hippocampal synaptic plasticity. Synapse 35, 151-159. https://doi.org/10.1002/(SICI)1098-2396(200002)35:2<151::AID-SYN8>3.0.CO;2-P

Altamura, M., Caradonna, L., Amati, L., Pellegrino, N.M., Urgesi, G., Miniello, S., 2001. Splenectomy and sepsis: The role of the spleen in the immune-mediated bacterial clearance. Immunopharmacol. Immunotoxicol. 23, 153-161. https://doi.org/10.1081/IPH-100103856

Andrade, R.M., Portillo, J.A.C., Wessendarp, M., Subauste, C.S., 2005. CD40 signaling in macrophages induces activity against an intracellular pathogen independently of gamma interferon and reactive nitrogen intermediates. Infect. Immun. 73, 3115-3123. https://doi.org/10.1128/IAI.73.5.3115-3123.2005

Annamneedi, A., Caliskan, G., Müller, S., Montag, D., Budinger, E., Angenstein, F., Fejtova, A., Tischmeyer, W., Gundelfinger, E.D., Stork, O., 2018. Ablation of the presynaptic organizer Bassoon in excitatory neurons retards dentate gyrus maturation and enhances learning performance. Brain Struct. Funct. 223, 3423-3445. https://doi.org/10.1007/s00429-018-1692-3

Barbeito, L.H., Pehar, M., Cassina, P., Vargas, M.R., Peluffo, H., Viera, L., Estévez, A.G., Beckman, J.S., 2004. A role for astrocytes in motor neuron loss in amyotrophic lateral sclerosis. Brain Res. Rev. 47, 263-274. https://doi.org/10.1016/j.brainresrev.2004.05.003

Baruch, K., Rosenzweig, N., Kertser, A., Deczkowska, A., Sharif, A.M., Spinrad, A., TsitsouKampeli, A., Sarel, A., Cahalon, L., Schwartz, M., 2015. Breaking immune tolerance by targeting Foxp3+ regulatory T cells mitigates Alzheimer's disease pathology. Nat. Commun. 6. https://doi.org/10.1038/ncomms8967

Beattie, E.C., Stellwagen, D., Morishita, W., Bresnahan, J.C., Ha, B.K., Zastrow, M. Von, Beattie, M.S., Malenka, R.C., 2002. Control of Synaptic Strength by Glial TNFalpha. Science (80-. ). 295, 2282-2285. https://doi.org/10.1126/science.1067859

Bercik, P., Collins, S.M., 2014. The Effects of Inflammation, Infection and Antibiotics on the Microbiota-Gut-Brain Axis BT - Microbial Endocrinology: The Microbiota-Gut-Brain Axis in Health and Disease. Microb. Endocrinol. Microbiota-Gut-Brain Axis Heal. Dis. 279- 
289. https://doi.org/10.1007/978-1-4939-0897-4

Biswas, A., Bruder, D., Wolf, S.A., Jeron, A., Mack, M., Heimesaat, M.M., Dunay, I.R., 2015. Ly6C high Monocytes Control Cerebral Toxoplasmosis . J. Immunol. 194, 3223-3235. https://doi.org/10.4049/jimmunol.1402037

Buzsaki, G., 2004. Neuronal Oscillations in Cortical Networks. Science (80-. ). 304, 19261929. https://doi.org/10.1126/science. 1099745

Buzsáki, G., 2015. Hippocampal sharp wave-ripple: A cognitive biomarker for episodic memory and planning. Hippocampus 25, 1073-1188. https://doi.org/10.1002/hipo.22488

Çalişkan, G., Müller, I., Semtner, M., Winkelmann, A., Raza, A.S., Hollnagel, J.O., Rösler, A., Heinemann, U., Stork, O., Meier, J.C., 2016. Identification of Parvalbumin Interneurons as Cellular Substrate of Fear Memory Persistence. Cereb. Cortex 26, 2325-2340. https://doi.org/10.1093/cercor/bhw001

Caliskan, G., Schulz, S.B., Gruber, D., Behr, J., Heinemann, U., Gerevich, Z., 2015. Corticosterone and corticotropin-releasing factor acutely facilitate gamma oscillations in the hippocampus in vitro. Eur. J. Neurosci. 41, 31-44. https://doi.org/10.1111/ejn.12750

Çalışkan, G., Müller, I., Semtner, M., Winkelmann, A., Raza, A.S., Hollnagel, J.O., Rösler, A., Heinemann, U., Stork, O., Meier, J.C., 2016. Identification of Parvalbumin Interneurons as Cellular Substrate of Fear Memory Persistence. Cereb. Cortex 26, 2325-2340. https://doi.org/10.1093/cercor/bhw001

Çalışkan, G., Raza, S.A., Demiray, Y.E., Kul, E., Sandhu, K. V., Stork, O., 2019. Depletion of dietary phytoestrogens reduces hippocampal plasticity and contextual fear memory stability in adult male mouse. Nutr. Neurosci. 1-12. https://doi.org/10.1080/1028415X.2019.1698826

Çalışkan, G., Stork, O., 2019. Hippocampal network oscillations at the interplay between innate anxiety and learned fear. Psychopharmacology (Berl). 236, 321-338. https://doi.org/10.1007/s00213-018-5109-z

Çalışkan, G., Stork, O., 2018. Hippocampal network oscillations as mediators of behavioural metaplasticity: Insights from emotional learning. Neurobiol. Learn. Mem. 154, 37-53. https://doi.org/10.1016/j.nlm.2018.02.022

Cardona, A.E., Pioro, E.P., Sasse, M.E., Kostenko, V., Cardona, S.M., Dijkstra, I.M., Huang, D.R., Kidd, G., Dombrowski, S., Dutta, R., Lee, J.C., Cook, D.N., Jung, S., Lira, S.A., Littman, D.R., Ransohoff, R.M., 2006. Control of microglial neurotoxicity by the fractalkine receptor. Nat. Neurosci. 9, 917-924. https://doi.org/10.1038/nn1715

Chater, T.E., Goda, Y., 2014. The role of AMPA receptors in postsynaptic mechanisms of 
synaptic plasticity. Front. Cell. Neurosci. 8, 1-14.

https://doi.org/10.3389/fncel.2014.00401

Chu, C., Murdock, M.H., Jing, D., Won, T.H., Chung, H., Kressel, A.M., Tsaava, T., Addorisio, M.E., Putzel, G.G., Zhou, L., Bessman, N.J., Yang, R., Moriyama, S., Parkhurst, C.N., Li, A., Meyer, H.C., Teng, F., Chavan, S.S., Tracey, K.J., Regev, A., Schroeder, F.C., Lee, F.S., Liston, C., Artis, D., 2019. The microbiota regulate neuronal function and fear extinction learning. Nature 574, 543-548. https://doi.org/10.1038/s41586-019-1644-y

Citri, A., Malenka, R.C., 2008. Synaptic plasticity: Multiple forms, functions, and mechanisms. Neuropsychopharmacology 33, 18-41. https://doi.org/10.1038/sj.npp.1301559

Cobb, J.P., Laramie, J.M., Stormo, G.D., Morrissey, J.J., Shannon, W.D., Qiu, Y., Karl, I.E., Buchman, T.G., Hotchkiss, R.S., 2002. Sepsis gene expression profiling: Murine splenic compared with hepatic responses determined by using complementary DNA microarrays. Crit. Care Med. 30, 2711-2721. https://doi.org/10.1097/00003246200212000-00016

Conner, J.M., Franks, K.M., Titterness, A.K., Russell, K., Merrill, D.A., Christie, B.R., Sejnowski, T.J., Tuszynski, M.H., 2009. NGF is essential for hippocampal plasticity and learning. J. Neurosci. 29, 10883-10889. https://doi.org/10.1523/JNEUROSCI.259409.2009

Connor, S.A., Hoeffer, C.A., Klann, E., Nguyen, P. V., 2011. Fragile X mental retardation protein regulates heterosynaptic plasticity in the hippocampus. Learn. Mem. 18, 207220. https://doi.org/10.1101//m.2043811

Cryan, J.F., Dinan, T.G., 2015. Gut microbiota: Microbiota and neuroimmune signalling Metchnikoff to microglia. Nat. Rev. Gastroenterol. Hepatol. 12, 494-496. https://doi.org/10.1038/nrgastro.2015.127

Curran, B.P., O'Connor, J.J., 2003. The inhibition of long-term potentiation in the rat dentate gyrus by pro-inflammatory cytokines is attenuated in the presence of nicotine. Neurosci. Lett. 344, 103-106. https://doi.org/10.1016/S0304-3940(03)00440-3

Darch, H.T., Collins, M.K., O’Riordan, K.J., Cryan, J.F., 2021. Microbial memories: Sexdependent impact of the gut microbiome on hippocampal plasticity. Eur. J. Neurosci. https://doi.org/10.1111/ejn.15119

Desbonnet, L., Clarke, G., Traplin, A., O'Sullivan, O., Crispie, F., Moloney, R.D., Cotter, P.D., Dinan, T.G., Cryan, J.F., 2015. Gut microbiota depletion from early adolescence in mice: Implications for brain and behaviour. Brain. Behav. Immun. 48, 165-173. 
https://doi.org/10.1016/j.bbi.2015.04.004

Dröge, W., Schipper, H.M., 2007. Oxidative stress and aberrant signaling in aging and cognitive decline. Aging Cell 6, 361-370. https://doi.org/10.1111/j.1474-

9726.2007.00294.x

Dugan, L.L., Ali, S.S., Shekhtman, G., Roberts, A.J., Lucero, J., Quick, K.L., Behrens, M.M., 2009. IL-6 Mediated Degeneration of Forebrain GABAergic Interneurons and Cognitive Impairment in Aged Mice through Activation of Neuronal NADPH Oxidase. PLoS One 4, e5518. https://doi.org/10.1371/journal.pone.0005518

Düsedau, H.P., Kleveman, J., Figueiredo, C.A., Biswas, A., Steffen, J., Kliche, S., Haak, S., Zagrebelsky, M., Korte, M., Dunay, I.R., 2019. p75 NTR regulates brain mononuclear cell function and neuronal structure in Toxoplasma infection-induced neuroinflammation. Glia 67, 193-211. https://doi.org/10.1002/glia.23553

Etter, G., van der Veldt, S., Manseau, F., Zarrinkoub, I., Trillaud-Doppia, E., Williams, S., 2019. Optogenetic gamma stimulation rescues memory impairments in an Alzheimer's disease mouse model. Nat. Commun. 10, 5322. https://doi.org/10.1038/s41467-019$13260-9$

Fanselow, M.S., Dong, H.W., 2010. Are the Dorsal and Ventral Hippocampus Functionally Distinct Structures? Neuron 65, 7-19. https://doi.org/10.1016/j.neuron.2009.11.031

Fell, J., Axmacher, N., 2011. The role of phase synchronization in memory processes. Nat. Rev. Neurosci. 12, 105-118. https://doi.org/10.1038/nrn2979

Fisahn, A., Pike, F.G., Buhl, E.H., Paulsen, O., 1998. Cholinergic induction of network oscillations at $40 \mathrm{~Hz}$ in the hippocampus in vitro. Nature 394, 186-189. https://doi.org/10.1038/28179

French, T., Düsedau, H.P., Steffen, J., Biswas, A., Ahmed, N., Hartmann, S., Schüler, T., Schott, B.H., Dunay, I.R., 2019. Neuronal impairment following chronic Toxoplasma gondii infection is aggravated by intestinal nematode challenge in an IFN- $\gamma$-dependent manner. J. Neuroinflammation 16, 1-18. https://doi.org/10.1186/s12974-019-1539-8

Fröhlich, E.E., Farzi, A., Mayerhofer, R., Reichmann, F., Jačan, A., Wagner, B., Zinser, E., Bordag, N., Magnes, C., Fröhlich, E., Kashofer, K., Gorkiewicz, G., Holzer, P., 2016a. Cognitive impairment by antibiotic-induced gut dysbiosis: Analysis of gut microbiotabrain communication. Brain. Behav. Immun. 56, 140-155.

https://doi.org/10.1016/j.bbi.2016.02.020

Fröhlich, E.E., Farzi, A., Mayerhofer, R., Reichmann, F., Jačan, A., Wagner, B., Zinser, E., Bordag, N., Magnes, C., Fröhlich, E., Kashofer, K., Gorkiewicz, G., Holzer, P., 2016b. 
Cognitive impairment by antibiotic-induced gut dysbiosis: Analysis of gut microbiotabrain communication. Brain. Behav. Immun. 56, 140-155.

https://doi.org/10.1016/j.bbi.2016.02.020

Galic, M.A., Riazi, K., Pittman, Q.J., 2012. Cytokines and brain excitability. Front. Neuroendocrinol. 33, 116-125. https://doi.org/10.1016/j.yfrne.2011.12.002

Ge, X., Ding, C., Zhao, W., Xu, L., Tian, H., Gong, J., Zhu, M., Li, J., Li, N., 2017. Antibioticsinduced depletion of mice microbiota induces changes in host serotonin biosynthesis and intestinal motility. J. Transl. Med. 15, 1-9. https://doi.org/10.1186/s12967-016-11054

Girardeau, G., Benchenane, K., Wiener, S.I., Buzsáki, G., Zugaro, M.B., 2009. Selective suppression of hippocampal ripples impairs spatial memory. Nat. Neurosci. 12, 12221223. https://doi.org/10.1038/nn.2384

Girardeau, G., Inema, I., Buzsáki, G., 2017. Reactivations of emotional memory in the hippocampus-amygdala system during sleep. Nat. Neurosci. 20, 1634-1642. https://doi.org/10.1038/nn.4637

Girardeau, G., Zugaro, M., 2011. Hippocampal ripples and memory consolidation. Curr. Opin. Neurobiol. 21, 452-459. https://doi.org/10.1016/j.conb.2011.02.005

Hammond, M.D., Taylor, R.A., Mullen, M.T., Ai, Y., Aguila, H.L., Mack, M., Kasner, S.E., McCullough, L.D., Sansing, L.H., 2014. CCR2+Ly6Chi inflammatory monocyte recruitment exacerbates acute disability following intracerebral hemorrhage. J. Neurosci. 34, 3901-3909. https://doi.org/10.1523/JNEUROSCI.4070-13.2014

Hasam-Henderson, L.A., Gotti, G.C., Mishto, M., Klisch, C., Gerevich, Z., Geiger, J.R.P., Kovács, R., 2018. NMDA-receptor inhibition and oxidative stress during hippocampal maturation differentially alter parvalbumin expression and gamma-band activity. Sci. Rep. 8, 9545. https://doi.org/10.1038/s41598-018-27830-2

Headley, D.B., Paré, D., 2013. In sync: gamma oscillations and emotional memory. Front. Behav. Neurosci. 7, 170. https://doi.org/10.3389/fnbeh.2013.00170

Heijtz, R.D., Wang, S., Anuar, F., Qian, Y., Björkholm, B., Samuelsson, A., Hibberd, M.L., Forssberg, H., Pettersson, S., 2011. Normal gut microbiota modulates brain development and behavior. Proc. Natl. Acad. Sci. U. S. A. 108, 3047-3052. https://doi.org/10.1073/pnas.1010529108

Hollnagel, J.O., ul Haq, R., Behrens, C.J., Maslarova, A., Mody, I., Heinemann, U., 2015. No evidence for role of extracellular choline-acetyltransferase in generation of gamma oscillations in rat hippocampal slices in vitro. Neuroscience $284,459-469$. 
https://doi.org/10.1016/j.neuroscience.2014.10.016

Hoshino, K., Hasegawa, K., Kamiya, H., Morimoto, Y., 2017. Synapse-specific effects of IL$1 \beta$ on long-term potentiation in the mouse hippocampus. Biomed. Res. 38, 183-188. https://doi.org/10.2220/biomedres.38.183

laccarino, H.F., Singer, A.C., Martorell, A.J., Rudenko, A., Gao, F., Gillingham, T.Z., Mathys, H., Seo, J., Kritskiy, O., Abdurrob, F., Adaikkan, C., Canter, R.G., Rueda, R., Brown, E.N., Boyden, E.S., Tsai, L.H., 2016. Gamma frequency entrainment attenuates amyloid load and modifies microglia. Nature 540, 230-235. https://doi.org/10.1038/nature20587

Irwin, M.R., Miller, A.H., 2007. Depressive disorders and immunity: 20 years of progress and discovery. Brain. Behav. Immun. 21, 374-383. https://doi.org/10.1016/j.bbi.2007.01.010

Josefsdottir, K.S., Baldridge, M.T., Kadmon, C.S., King, K.Y., 2017. Antibiotics impair murine hematopoiesis by depleting the intestinal microbiota. Blood 129, 729-739.

https://doi.org/10.1182/blood-2016-03-708594

Kann, O., Huchzermeyer, C., Kovács, R., Wirtz, S., Schuelke, M., 2011. Gamma oscillations in the hippocampus require high complex i gene expression and strong functional performance of mitochondria. Brain 134, 345-358. https://doi.org/10.1093/brain/awq333

Kjelstrup, K.G., Tuvnes, F.A., Steffenach, H.-A., Murison, R., Moser, E.I., Moser, M.-B., 2002. Reduced fear expression after lesions of the ventral hippocampus. Proc. Natl. Acad. Sci. 99, 10825-10830. https://doi.org/10.1073/pnas.152112399

Knoop, K.A., McDonald, K.G., Kulkarni, D.H., Newberry, R.D., 2016. Antibiotics promote inflammation through the translocation of native commensal colonic bacteria. Gut 65 , 1100-1109. https://doi.org/10.1136/gutjnl-2014-309059

Lang, D., Schott, B.H., van Ham, M., Morton, L., Kulikovskaja, L., Herrera-Molina, R., Pielot, R., Klawonn, F., Montag, D., Jänsch, L., Gundelfinger, E.D., Smalla, K.H., Dunay, I.R., 2018. Chronic Toxoplasma infection is associated with distinct alterations in the synaptic protein composition. J. Neuroinflammation 15, 1-19. https://doi.org/10.1186/s12974018-1242-1

Leal, G., Bramham, C.R., Duarte, C.B., 2017. BDNF and Hippocampal Synaptic Plasticity, in: Vitamins and Hormones. Elsevier Inc., pp. 153-195.

https://doi.org/10.1016/bs.vh.2016.10.004

Li, D., Liu, X., Liu, T., Liu, H., Tong, L., Jia, S., Wang, Y.F., 2020. Neurochemical regulation of the expression and function of glial fibrillary acidic protein in astrocytes. Glia $68,878-$ 897. https://doi.org/10.1002/glia.23734

Lyons, A., Lynch, A.M., Downer, E.J., Hanley, R., O’Sullivan, J.B., Smith, A., Lynch, M.A., 
2009. Fractalkine-induced activation of the phosphatidylinositol-3 kinase pathway attentuates microglial activation in vivo and in vitro. J. Neurochem. 110, 1547-1556. https://doi.org/10.1111/j.1471-4159.2009.06253.x

Maggio, N., Segal, M., 2011. Persistent changes in ability to express long-term potentiation/depression in the rat hippocampus after juvenile/adult stress. Biol. Psychiatry 69, 748-753. https://doi.org/10.1016/j.biopsych.2010.11.026

Maier, N., Nimmrich, V., Draguhn, A., 2003. Cellular and network mechanisms underlying spontaneous sharp wave-ripple complexes in mouse hippocampal slices. J. Physiol. 550, 873-887. https://doi.org/10.1113/jphysiol.2003.044602

Mander, P., Brown, G.C., 2005. Activation of microglial NADPH oxidase is synergistic with glial iNOS expression in inducing neuronal death: A dual-key mechanism of inflammatory neurodegeneration. J. Neuroinflammation 2, 1-15. https://doi.org/10.1186/1742-2094-2-20

Maren, S., Phan, K.L., Liberzon, I., 2013. The contextual brain: implications for fear conditioning, extinction and psychopathology. Nat. Rev. Neurosci. 14, 417-428. https://doi.org/10.1038/nrn3492

Martorell, A.J., Paulson, A.L., Suk, H.J., Abdurrob, F., Drummond, G.T., Guan, W., Young, J.Z., Kim, D.N.W., Kritskiy, O., Barker, S.J., Mangena, V., Prince, S.M., Brown, E.N., Chung, K., Boyden, E.S., Singer, A.C., Tsai, L.H., 2019. Multi-sensory Gamma Stimulation Ameliorates Alzheimer's-Associated Pathology and Improves Cognition. Cell 177, 256-271.e22. https://doi.org/10.1016/j.cell.2019.02.014

Mayer, E.A., 2011. Gut feelings: The emerging biology of gut-"brain communication. Nat. Rev. Neurosci. 12, 453-466. https://doi.org/10.1038/nrn3071

McCoy, M.K., Tansey, M.G., 2008. TNF signaling inhibition in the CNS: Implications for normal brain function and neurodegenerative disease. J. Neuroinflammation 5, 1-13. https://doi.org/10.1186/1742-2094-5-45

Michell-Robinson, M.A., Touil, H., Healy, L.M., Owen, D.R., Durafourt, B.A., Bar-Or, A., Antel, J.P., Moore, C.S., 2015. Roles of microglia in brain development, tissue maintenance and repair. Brain 138, 1138-1159. https://doi.org/10.1093/brain/awv066

Möhle, L., Israel, N., Paarmann, K., Krohn, M., Pietkiewicz, S., Müller, A., Lavrik, I.N., Buguliskis, J.S., Schott, B.H., Schlüter, D., Gundelfinger, E.D., Montag, D., Seifert, U., Pahnke, J., Dunay, I.R., 2016a. Chronic Toxoplasma gondii infection enhances $\beta$ amyloid phagocytosis and clearance by recruited monocytes. Acta Neuropathol. Commun. 4, 25. https://doi.org/10.1186/s40478-016-0293-8 
Möhle, L., Mattei, D., Heimesaat, M.M., Bereswill, S., Fischer, A., Alutis, M., French, T., Hambardzumyan, D., Matzinger, P., Dunay, I.R., Wolf, S.A., 2016b. Ly6Chi Monocytes Provide a Link between Antibiotic-Induced Changes in Gut Microbiota and Adult Hippocampal Neurogenesis. Cell Rep. 15, 1945-1956. https://doi.org/10.1016/j.celrep.2016.04.074

Montgomery, S.M., Buzsaki, G., 2007. Gamma oscillations dynamically couple hippocampal CA3 and CA1 regions during memory task performance. Proc. Natl. Acad. Sci. 104, 14495-14500. https://doi.org/10.1073/pnas.0701826104

Mostafa, S., Miller, B.J., 2014. Antibiotic-associated psychosis during treatment of urinary tract infections: A systematic review. J. Clin. Psychopharmacol. 34, 483-490. https://doi.org/10.1097/JCP.0000000000000150

Nash, K.R., Moran, P., Finneran, D.J., Hudson, C., Robinson, J., Morgan, D., Bickford, P.C., 2015. Fractalkine over expression suppresses a-synuclein-mediated neurodegeneration. Mol. Ther. 23, 17-23. https://doi.org/10.1038/mt.2014.175

Neufeld, K.M., Kang, N., Bienenstock, J., Foster, J.A., 2011. Reduced anxiety-like behavior and central neurochemical change in germ-free mice. Neurogastroenterol. Motil. 23, 255-265. https://doi.org/10.1111/j.1365-2982.2010.01620.x

Ogawa, Y., Miyoshi, C., Obana, N., Yajima, K., Hotta-Hirashima, N., Ikkyu, A., Kanno, S., Soga, T., Fukuda, S., Yanagisawa, M., 2020. Gut microbiota depletion by chronic antibiotic treatment alters the sleep/wake architecture and sleep EEG power spectra in mice. Sci. Rep. 10, 1-11. https://doi.org/10.1038/s41598-020-76562-9

Okuno, T., Nakatsuji, Y., Kumanogoh, A., Moriya, M., Ichinose, H., Sumi, H., Fujimura, H., Kikutani, H., Sakoda, S., 2005. Loss of dopaminergic neurons by the induction of inducible nitric oxide synthase and cyclooxygenase-2 via CD40: Relevance to Parkinson's disease. J. Neurosci. Res. 81, 874-882. https://doi.org/10.1002/jnr.20599

Papageorgiou, I.E., Lewen, A., Galow, L. V., Cesetti, T., Scheffel, J., Regen, T., Hanisch, U.K., Kann, O., 2016. TLR4-activated microglia require IFN- $y$ to induce severe neuronal dysfunction and death in situ. Proc. Natl. Acad. Sci. U. S. A. 113, 212-217. https://doi.org/10.1073/pnas.1513853113

Perry, S.W., Dewhurst, S., Bellizzi, M.J., Gelbard, H.A., 2002. Tumor Necrosis Factor-Alpha in Normal and Diseased Brain: Conflicting Effects Via Intraneuronal Receptor Crosstalk? J. Neurovirol. 8, 611-624. https://doi.org/10.1080/13550280290101021

Polepalli, J.S., Wu, H., Goswami, D., Halpern, C.H., Südhof, T.C., Malenka, R.C., 2017. Modulation of excitation on parvalbumin interneurons by neuroligin-3 regulates the 
hippocampal network. Nat. Neurosci. 20, 219-229. https://doi.org/10.1038/nn.4471

Probert, L., 2015. TNF and its receptors in the CNS: The essential, the desirable and the deleterious effects. Neuroscience 302, 2-22.

https://doi.org/10.1016/j.neuroscience.2015.06.038

Ramalingam, M., Kim, S.-J., 2012. Reactive oxygen/nitrogen species and their functional correlations in neurodegenerative diseases. J. Neural Transm. 119, 891-910. https://doi.org/10.1007/s00702-011-0758-7

Rogers, J.T., Morganti, J.M., Bachstetter, A.D., Hudson, C.E., Peters, M.M., Grimmig, B.A., Weeber, E.J., Bickford, P.C., Gemma, C., 2011. CX3CR1 deficiency leads to impairment of hippocampal cognitive function and synaptic plasticity. J. Neurosci. 31, 16241-16250. https://doi.org/10.1523/JNEUROSCI.3667-11.2011

Rowan, M.J., Klyubin, I., Cullen, W.K., Anwyl, R., 2003. Synaptic plasticity in animal models of early Alzheimer's disease. Philos. Trans. R. Soc. B Biol. Sci. 358, 821-828. https://doi.org/10.1098/rstb.2002.1240

Sarkar, A., Harty, S., Lehto, S.M., Moeller, A.H., Dinan, T.G., Dunbar, R.I.M., Cryan, J.F., Burnet, P.W.J., 2018. The Microbiome in Psychology and Cognitive Neuroscience. Trends Cogn. Sci. 22, 611-636. https://doi.org/10.1016/j.tics.2018.04.006

Sarkar, S.R., Mazumder, P.M., Banerjee, S., 2020. Probiotics protect against gut dysbiosis associated decline in learning and memory. J. Neuroimmunol. 348, 577390. https://doi.org/10.1016/j.jneuroim.2020.577390

Schwartz, M., Kipnis, J., Rivest, S., Prat, A., 2013. How do immune cells support and shape the brain in health, disease, and aging? J. Neurosci. 33, 17587-17596. https://doi.org/10.1523/JNEUROSCI.3241-13.2013

Sesti, F., Liu, S., Cai, S.-Q., 2010. Oxidation of potassium channels by ROS: a general mechanism of aging and neurodegeneration? Trends Cell Biol. 20, 45-51. https://doi.org/10.1016/j.tcb.2009.09.008

Shechter, R., Schwartz, M., 2013. Harnessing monocyte-derived macrophages to control central nervous system pathologies: No longer if' but how'. J. Pathol. 229, 332-346. https://doi.org/10.1002/path.4106

Shi, C., Pamer, E.G., 2011. Monocyte recruitment during infection and inflammation. Nat. Rev. Immunol. 11, 762-774. https://doi.org/10.1038/nri3070

Sica, A., Mantovani, A., 2012. Macrophage plasticity and polarization: in vivo veritas. J. Clin. Invest. 122, 787-795. https://doi.org/10.1172/JCI59643 
Stellwagen, D., Malenka, R.C., 2006. Synaptic scaling mediated by glial TNF- $\alpha$. Nature 440, 1054-1059. https://doi.org/10.1038/nature04671

Straub, R.H., Linde, H.J., Männel, D.N., Schölmerich, J., Falk, W., 2000. A bacteria-induced switch of sympathetic effector mechanisms augments local inhibition of TNF-alpha and IL-6 secretion in the spleen. FASEB J. 14, 1380-1388.

https://doi.org/10.1096/fj.14.10.1380

Suda, K.J., Hicks, L.A., Roberts, R.M., Hunkler, R.J., Taylor, T.H., 2014. Trends and Seasonal Variation in Outpatient Antibiotic Prescription Rates in the United States, 2006 to 2010. Antimicrob. Agents Chemother. 58, 2763-2766.

https://doi.org/10.1128/AAC.02239-13

Sun, N., Hu, H., Wang, F., Li, L., Zhu, W., Shen, Y., Xiu, J., Xu, Q., 2021. Antibiotic-induced microbiome depletion in adult mice disrupts blood-brain barrier and facilitates brain infiltration of monocytes after bone-marrow transplantation. Brain. Behav. Immun. 92, 102-114. https://doi.org/10.1016/j.bbi.2020.11.032

Ta, T.T., Dikmen, H.O., Schilling, S., Chausse, B., Lewen, A., Hollnagel, J.O., Kann, O., 2019. Priming of microglia with IFN-y slows neuronal gamma oscillations in situ. Proc. Natl. Acad. Sci. U. S. A. 116, 4637-4642. https://doi.org/10.1073/pnas.1813562116

Trimper, J.B., Stefanescu, R.A., Manns, J.R., 2014. Recognition memory and theta-gamma interactions in the hippocampus. Hippocampus 24, 341-353.

https://doi.org/10.1002/hipo.22228

Vandecasteele, M., Varga, V., Berényi, A., Papp, E., Barthó, P., Venance, L., Freund, T.F., Buzsáki, G., 2014. Optogenetic activation of septal cholinergic neurons suppresses sharp wave ripples and enhances theta oscillations in the hippocampus. Proc. Natl. Acad. Sci. U. S. A. 111, 13535-40. https://doi.org/10.1073/pnas.1411233111

Wolf, Y., Yona, S., Kim, K.W., Jung, S., 2013. Microglia, seen from the CX3CR1 angle. Front. Cell. Neurosci. 7, 1-9. https://doi.org/10.3389/fncel.2013.00026

Zhan, Y., Paolicelli, R.C., Sforazzini, F., Weinhard, L., Bolasco, G., Pagani, F., Vyssotski, A.L., Bifone, A., Gozzi, A., Ragozzino, D., Gross, C.T., 2014. Deficient neuron-microglia signaling results in impaired functional brain connectivity and social behavior. Nat.

Neurosci. 17, 400-406. https://doi.org/10.1038/nn.3641 